ARTICLE

https://doi.org/10.1038/s41467-019-13001-y

\title{
Invariant patterns of clonal succession determine specific clinical features of myelodysplastic syndromes
}

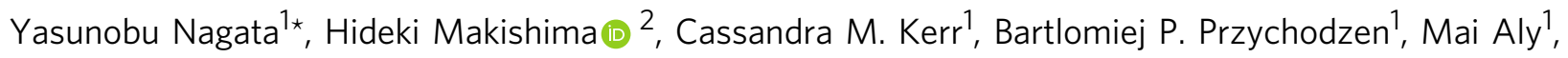
Abhinav Goyal ${ }^{1}$, Hassan Awada', Mohammad Fahad Asad ${ }^{1}$, Teodora Kuzmanovic $^{1}$, Hiromichi Suzuki ${ }^{2}$, Tetsuichi Yoshizato ${ }^{2}$, Kenichi Yoshida², Kenichi Chiba ${ }^{3}$, Hiroko Tanaka (10 ${ }^{3}$, Yuichi Shiraishi ${ }^{3}$, Satoru Miyano ${ }^{3}$, Sudipto Mukherjee ${ }^{4}$, Thomas LaFramboise ${ }^{5}$, Aziz Nazha4, Mikkael A. Sekeres ${ }^{4}$, Tomas Radivoyevitch (1) ${ }^{1}$, Torsten Haferlach ${ }^{6}$, Seishi Ogawa ${ }^{2} \&$ Jaroslaw P. Maciejewski ${ }^{1 \star}$

Myelodysplastic syndromes (MDS) arise in older adults through stepwise acquisitions of multiple somatic mutations. Here, analyzing 1809 MDS patients, we infer clonal architecture by using a stringent, the single-cell sequencing validated PyClone bioanalytic pipeline, and assess the position of the mutations within the clonal architecture. All 3,971 mutations are grouped based on their rank in the deduced clonal hierarchy (dominant and secondary). We evaluated how they affect the resultant morphology, progression, survival and response to therapies. Mutations of SF3B1, U2AF1, and TP53 are more likely to be dominant, those of $A S X L 1, C B L$, and KRAS are secondary. Among distinct combinations of dominant/secondary mutations we identified 37 significant relationships, of which 12 affect clinical phenotypes, 5 cooperatively associate with poor prognosis. They also predict response to hypomethylating therapies. The clonal hierarchy has distinct ranking and the resultant invariant combinations of dominant/secondary mutations yield novel insights into the specific clinical phenotype of MDS.

\footnotetext{
${ }^{1}$ Department of Translational Hematology \& Oncology Research, Taussig Cancer Institute, Cleveland Clinic, Cleveland, OH, USA. ${ }^{2}$ Department of Pathology and Tumor Biology, Graduate School of Medicine, Kyoto University, Kyoto, Japan. ${ }^{3}$ Laboratory of DNA Information Analysis, Human Genome Center, Institute of Medical Science, The University of Tokyo, Tokyo, Japan. ${ }^{4}$ Department of Hematology and Medical Oncology, Taussig Cancer Institute, Cleveland Clinic, Cleveland, OH, USA. ${ }^{5}$ Department ofGenetics and Genome Sciences, Case Western Reserve University, Cleveland, OH, USA. ${ }^{6}$ MLL Munich Leukemia Laboratory, Munich, Germany. *email: ysnagata-tky@umin.ac.jp; maciejj@ccf.org
} 
M yelodysplastic syndromes (MDS) are a clinically and molecularly heterogeneous collections of diseases ${ }^{1,2}$. They affect older adults and are characterized by dysplastic hematopoiesis, cytopenias, and a propensity for progression to acute myeloid leukemia (AML) ${ }^{3-6}$. Recent discoveries enabled by next-generation sequencing (NGS) have led to new insights into the pathogenic origins of MDS ${ }^{7-16}$.

Irrespective of origins, MDS pathogenesis includes initial ancestral lesion followed by the stepwise acquisition of subsequent somatic mutations resulting in a highly diverse clonal hierarchy ${ }^{17}$. While strong driver hits result in MDS instantly in form of a de novo disease, some of the founder mutations in MDS originate from subclinical clonal expansions, referred to as clonal hematopoiesis $(\mathrm{CH})$ present in the blood of some otherwise healthy individuals ${ }^{18,19}$. The prevalence of $\mathrm{CH}$ increases with age and is highly suggestive of a prodromal stage of MDS, i.e., $\mathrm{CH}$ may be a pre-MDS state. In some individuals, $\mathrm{CH}$ progresses to MDS via acquisition of secondary hits. However, due to competing risks of mortality and long latencies of subsequent transforming phenotypes, only a fraction of carriers with $\mathrm{CH}$ mutations develops $\mathrm{MDS}^{20-23}$. Initially, asymptomatic $\mathrm{CH}$ mutations are thus much less penetrant than typical AML hits, e.g. $\mathrm{t}(15 ; 17)$ or translocations involving $M L L^{24,25}$.

We hypothesized that partitioning patients based on ancestral and secondary hits would yield new metrics of MDS pathogenesis that are predictive of clinical and morphologic features and prognostic of outcomes. Heretofore, mapping mutation-state combinatorics to phenotypes have demonstrated a tremendous complexity, but have not yielded generalizable rules; correlations with classical morphologic subdivisions have been weak ${ }^{26}$. Clustering mutations by their rank in the clonal hierarchy or ancestral hit-deducted derivation as de novo or $\mathrm{CH}$-related disease may illuminate disease teleology and consequent clinical outcome. Fundamental to the interpretation of clonal hierarchy is determining whether ancestral hits are succeeded by random vs. predetermined secondary hits, and whether mutation interactions influence phenotypes. Primary hits may drive general phenotypes that are then modulated by secondary hits or alternatively, they may initiate leukemogenic processes and secondary hits may fully determine phenotypes and progression rates.

Clonal hierarchies are best characterized using deep sequencing on serial samples starting with $\mathrm{CH}$ and progressing through MDS to AML. Single-cell sequencing allows for cross-sectional analysis of clonal architecture and most precise recapitulation of clonal ontology $y^{2,27}$. Neither of these two approaches is suitable, though, to clinical realities: patients rarely have serial samples obtained, and routine single-cell sequencing of many patients is not yet feasible, certainly not for screenings for $\mathrm{CH}$ in healthy or in other large clinical cohorts. While single-cell investigation of individual patients can yield excellent results, broadly investigate this method to appropriately sized cohorts of patients precludes generalizable conclusions commensurate with the individual diversity. Consequently, the Beta Binomial emission model implemented in PyClone has been developed to recapitulate clonal hierarchy ${ }^{28}$. Limitations aside, these approaches provide hierarchical ranks of mutations that reflect clonal succession from primary/dominant hits to subsequent secondary hits.

Here, using a large cohort of patients and innovative analytical approaches, we investigated the origins of MDS and characterize the extent to which clonal succession rules exist and are predictive of MDS morphologic features and prognoses.

\section{Results}

Mutational profile of MDS. Our study included newly diagnosed and fully annotated 1809 patients with MDS or MDS/MPN

\section{Table 1 Demographic data of 1809 MDS patients subject to} sequencing.

\begin{tabular}{llll} 
Parameter & WES & TSa & Total cohort \\
\hline Patient numbers & 225 & 1584 & 1809 \\
$\begin{array}{l}\text { Median age } \\
\text { Ratio of male }\end{array}$ & 70.9 & 71.8 & 71.8 \\
$\begin{array}{l}\text { to female } \\
\text { Survival (median, } \\
\text { months) }\end{array}$ & 25 & 1.7 & 1.6 \\
$\begin{array}{l}\text { Diagnosis } \\
\text { MDS }\end{array}$ & $152(68 \%)$ & $1294(82 \%)$ & $1446(80 \%)$ \\
$\begin{array}{l}\text { MDS/MPN } \\
\text { SAMLb }\end{array}$ & $41(18 \%)$ & $171(11 \%)$ & $212(12 \%)$ \\
$\begin{array}{l}\text { Subtype of progression } \\
\text { Low riskc }\end{array}$ & $117(52 \%)$ & $119(7 \%)$ & $151(8 \%)$ \\
High riskc & $108(48 \%)$ & $658(41 \%)$ & $1043(58 \%)$ \\
\hline
\end{tabular}

WES whole-exome sequencing, TS targeted sequencing, MDS myelodysplastic syndromes, MPN myeloproliferative neoplasms, sAML secondary acute myeloid leukemia from MDS or MDS/ MPN

aln total, 36 common genes were sequenced in the entire cohort

bAll sAML cases were determined derived from MDS $(n=137)$ or MDS/MPM $(n=14)$

'Two groups based on IPSS-R scores (low risk as $<=3.5$ and high risk as $>3.5$ ) according to the transformation risk (Pfeilstöcker, M. et al. ${ }^{47}$ )

overlap, including lower- and higher-risk subtypes (LR or HR; Table 1 also see description of the patients in the Methods section) analyzed with clinical parameters and a panel of the most frequently mutated 36 myeloid genes using deep targeted NGS (TS) (Supplementary Table 1$)$. In a subset $(12 \%, n=225 / 1809)$ of these patients, whole-exome sequencing (WES) was performed to assess the congruence of the results with targeted sequencing (Supplementary Table 2). A total of 1169 patients from a previous report ${ }^{29}\left(52 \%\right.$ of the previous cohort ${ }^{29}$ ) were fully annotated by copy-number alterations, read counts of mutations, and uniformly applied morphologic assessment for inferring accurate clonal structure, which were included in this study. We also added 640 entirely new patients (Supplementary Fig. 1). Among 36 genes tested after removing SNPs/errors, 3971 somatic mutations were recorded and combined with copy-number alterations (CNA; Supplementary Figs. 2-5). The most frequently mutated genes/CNAs were TET2 (27\%), SF3B1 (23\%), ASXL1 (19\%), del(5q) (16\%), SRSF2 (14\%), DNMT3A (11\%), and $-7 /$ del7q (10\%), each present in $>10 \%$ of patients (Fig. 1a). Overall, 30 genetic hits (mutations/CNAs) were identified in $>2 \%$ of patients. We then examined the pairing likelihood of genomic lesions: 110 of the most common significantly associated mutant combinations were found (Supplementary Fig. 6 and Supplementary Data 1). To assess the impact of mutations on the phenotype, we then ranked them in two-dimensional space according to the odds of MDS vs. MDS/MPN association (history of MDS or MPN/MDS for the corresponding sAML) or LR vs. HR subtypes. For instance, del(5q), STAG2 mutations, and complex karyotype more likely associated with MDS features (Fig. 1b), while JAK2, EZH2, and RAS pathway (NRAS/KRAS/ $C B L)$ mutations associate with MDS/MPN overlap features. Similarly, RUNX1, STAG2 mutations, or $-7 / \operatorname{del}(7 \mathrm{q})$ were more likely to group with HR subtypes in contrast to $S F 3 B 1$, and JAK2 mutations predictive of LR subtypes.

Ancestral hits and recapitulation of clonal hierarchy. Typically, one of the mutational events initiates the subsequent cascade of subclonal events, resulting in clonal evolution. We hypothesized that most dominant mutations and subsequent hits can be ranked and assessed in such context irrespective of the nominal clonal burden. Thus, the impact of individual mutations may depend on their position within the clonal hierarchy with a special role for 
a

b
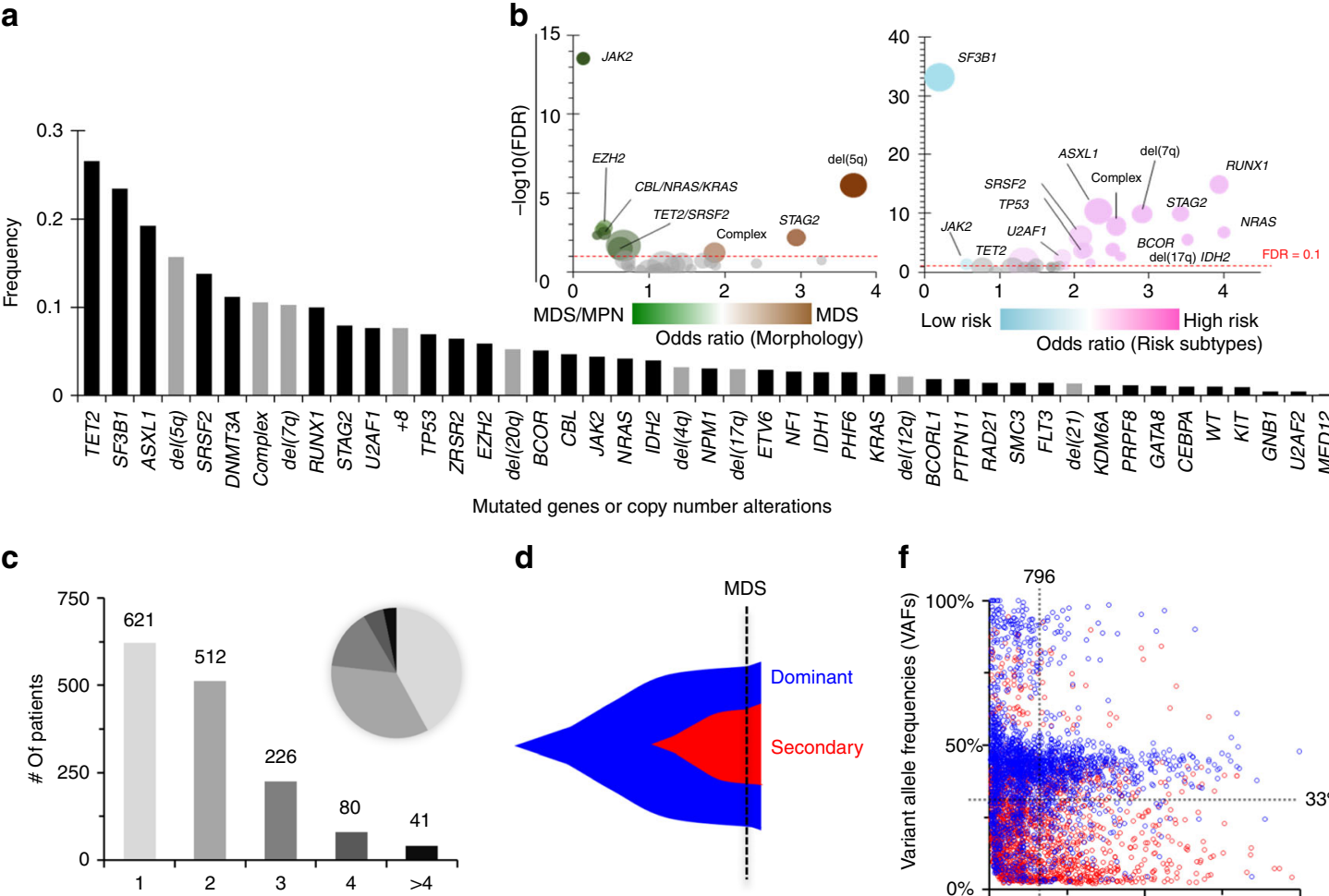

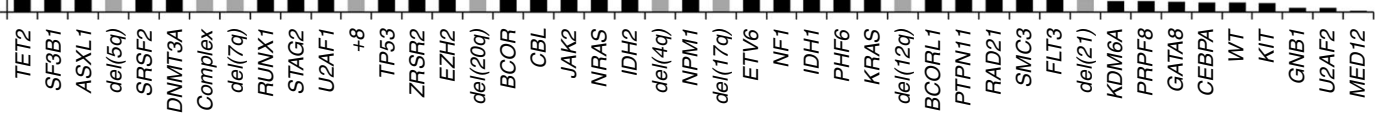

Mutated genes or copy number alterations

C

e

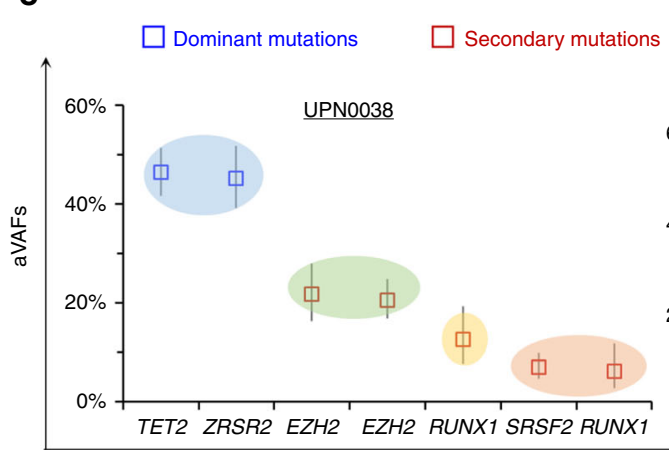

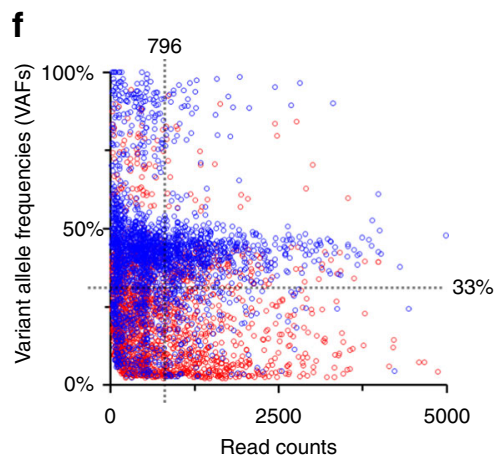

Different clones
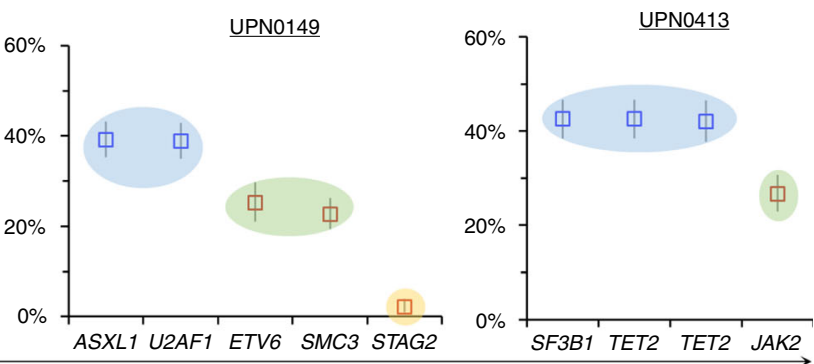

Mutated genes

g
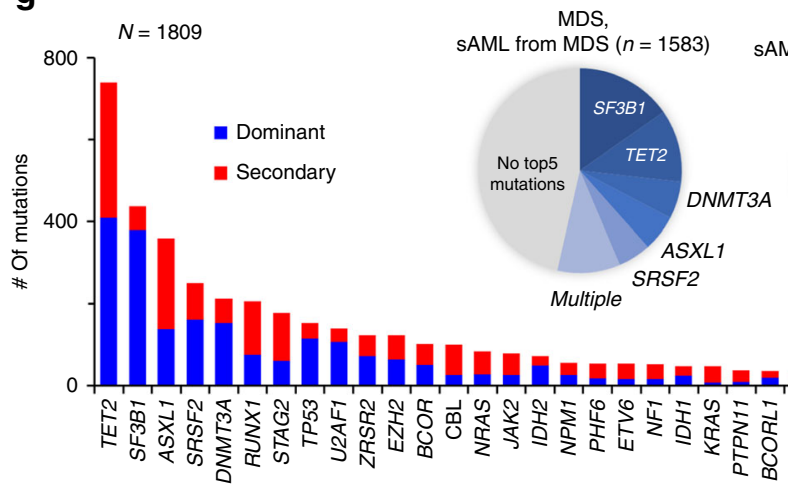

MDS/MPN,

AML from MDS/MPN $(n=226)$

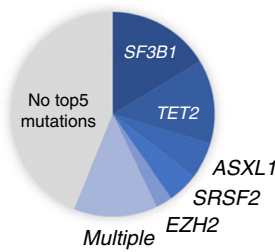

h

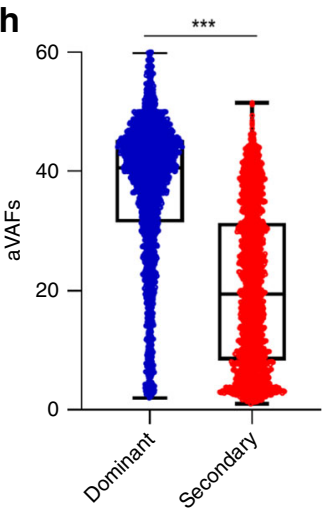

Genes

the earliest (ancestral or dominant) vs. secondary events. To identify significant differences between them, a stringent bioanalytic pipeline relying on differential clonal size in accordance with the read counts was employed. We investigated the temporal ranking of mutations during clonal evolution by the PyClone pipeline, which has been validated using single-cell sequencing ${ }^{28}$. It uses a Bayesian clustering method for grouping sets of deeply sequenced somatic mutations into putative clonal clusters, which revealed speculated clonal structure in our MDS patients, of which 859 patients harbored multiple (median 2, range 1-7) 
Fig. 1 Mutational landscapes, and dominant/secondary mutations. a Frequency of the 36 mutated genes (dark bars) and copy-number alterations (gray bars). $\mathbf{b}$ Clinical features associated with genetic hits. Effect of frequent genetic hits $(>2 \%, n=30$ ) were assessed. Bubble sizes encode the frequency of mutations in 1809 patients; odds ratios (OR) of MDS $(n=1583)$ vs. MDS/MPN $(n=226)$ and low- $(n=1043)$ vs. high risk $(n=766)$ are plotted on the $x$ axes. Negative log10 false discovery rates (FDR, i.e., $q$-values) are shown on the $y$-axes; our FDR cutoff of interest, 0.1 , is shown as a dashed horizontal red line. c The recent advanced algorithm called PyClone allowed the inference of clonal structure. All 3971 mutations, identified in 1809 samples, were evaluated in each sample. Multiple clones were found in 859 samples. The bar graphs and pie chart show the number and the fraction of samples with different number of clones, respectively. $\mathbf{d}$ Clonal evolution of MDS. Ancestral/initial hits were classified dominant, and subsequent/secondary hits were classified as secondary mutations. e Representative three samples with multiple clones. Copy-number-adjusted variant allele frequencies (aVAFs) ( $y$-axis) and mutated genes ( $x$-axis) in three illustrative samples are shown. Blue and red squares depict dominant and secondary mutations. Circles with different colors show the different clones estimated by PyClone ${ }^{28}$. f Sequencing read counts and raw VAFs. This scatter plot depicts 3971 mutations identified in 1809 patients. Blue and red bars indicate dominant and secondary mutations, respectively. Dotted vertical and horizontal lines depict mean total read counts and VAFs, respectively. $\mathbf{g}$ Distribution of dominant, co-dominant, and secondary mutations in all 36 genes in our panel. Dominant $(n=2155)$ and secondary mutations $(n=1816)$ are shown. Pie charts show the fraction of dominant mutations for each gene. The top five most frequently mutated genes are indicated in the left (MDS, sAML from MDS) and right (MDS/MPN, sAML form MDS/MPN), respectively. $\mathbf{h}$ Distribution of aVAFs between dominant vs. secondary mutations. Dot plots depict dominant (blue) and secondary (red) mutations. Box and whiskers indicate median and minimum to maximum of aVAFs for dominant and secondary mutations. ${ }^{\star \star \star} P<0.0001$ (Mann-Whitney's $U$ test)

(Fig. 1c). Clonal hierarchy was reduced to a one-dimensional ordered 2-level feature space of primary/dominant and secondary mutations (Fig. 1d). Mutations belonging to the largest clone were defined as dominant mutations, and other clones as secondary mutations (Supplementary Data 2). Several representative patients are shown (Fig. 1e; Supplementary Fig. 7). Mean variant allele frequencies (VAFs) and total read counts were $33 \%$ and 796, respectively (Fig. 1f). Also, dominant clone distribution, as determined by targeted panel, matched that of founder clones by WES, indicating that the targeted panel does reflect the spectrum of most important early mutations (Supplementary Fig. 8). Distribution of dominant and secondary mutations depended on each gene and morphologic features (Fig. 1g). In total, 2155 (54\%) band $1816(46 \%)$ mutations were judged to be dominant and secondary, respectively. Median VAFs of dominant and secondary mutations were 40.6 and $19.4 \%$ (Mann-Whitney's $U$ test; $P<$ 0.0001) (Fig. 1h). Mutations of SF3B1, U2AF1, TP53, DNMT3A, $I D H 2, S R S F 2$, and TET2 were more likely to be dominant, while those of $A S X L 1, J A K 2, C B L$, and KRAS were more likely to be secondary (Fig. 2a, b). For some genes, canonical vs. other missense or truncating mutations may have a different position within the clonal hierarchy. For instance, DNMT3A R882 mutations were more likely to be dominant compared with truncating or other missense mutations, which were more likely to be secondary (Supplementary Fig. 9). The top five dominant mutation genes were SF3B1, TET2, ASXL1, DNMT3A, and SRSF2 (Fig. 2c). TET2, ASXL1, and SRSF2 were also identified in secondary mutations (Fig. 2d).

Molecular association of dominant and secondary mutations. Our results suggest that, starting with the initial mutations, subsequent hits are not random, but rather follow a certain order (Fig. 2e), and that certain mutation combinations (dominant/ secondary) occur more commonly. Statistically significant correlations were found across 37 distinctive gene pairs (Fig. $2 \mathrm{f}$ and Table 2); 30 and 7 mutation pairs either coincided or were mutually exclusive, respectively. For example, TP53 dominant mutations were more likely to precede secondary TP53 mutations, but less likely secondary ASXL1 mutations (Supplementary Fig. 10). Similarly, dominant $I D H 2$ mutations preceded secondary ASXL1, SRSF2, and STAG2 mutations. Dominant TET2 mutations also preceded secondary TET2, and ZRSR2 mutations. While ASXL1 was commonly affected by specific secondary mutations, they occurred preferentially in the context of given dominant alterations. For instance, dominant SRSF2, U2AF1, EZH2, RUNX1, STAG2, IDH2, and CBL mutations were more likely to precede secondary ASXL1 mutations, and conversely dominant TP53, ASXL1, and SF3B1 mutations were less likely to co-occur secondary ASXL1 mutations (Supplementary Fig. 11).

Pairs of dominant and secondary mutations impact phenotypes. We then evaluated the impact of different types of mutations and their combinations on clinical phenotypes, including dichotomous morphological (MDS vs. MDS/MPN) features, progressive (low- vs. high risk) subtypes and survival. Depending on the position in the clonal hierarchy, the impact of specific mutations could vary in several genes (Supplementary Fig. 12). For instance, focusing on TET2 and/or SRSF2, these mutations were associated with MDS/MPN phenotypes and high-risk disease (likely CMML) (Fig. 3a). TET2 and SRSF2 mutations were enriched in CMML patients [44\% (53/121) and 32\% (39/121), respectively], and $20 \%(24 / 121)$ of the CMML patients had both TET2/SRSF2 mutations, a significantly frequent compared with the rest of MDS/MPN patients [2\% (2/91) of the MDSMPN-U/ RARS-T patients, $P<0.00001]$. With regard to the clonal architecture, patients with SRSF2 mutations as secondary hits were associated with MDS/MPN [OR $0.43(0.26-0.74), P=0.001]$. Given that patients with dominant or secondary TET2 mutations were unlikely to have MDS/MPN, one could hypothesize that phenotypic penetrance of SRSF2 mutations would likely be greater than that of TET2 mutations. In order to verify this hypothesis, we analyzed patients with both SRSF2 and TET2 mutations in detail (Fig. 3b). When patients with TET2 mutations acquired secondary SRSF2 mutations, they were significantly more likely to develop MDS/MPN [OR 0.19 (0.09-0.39), $P=$ $\left.2.7 \times 10^{-6}\right]$. Conversely, patients with SRSF2 mutations who had a secondary TET2 mutation had similar association with MDS/ MPN (SRSF2 followed by dominant TET2; OR 0.43 or vice versa SRSF2 followed by secondary TET2; OR 0.43). In additional analysis of specific pairs of mutations, patients with dominant TET2 followed by secondary SRSF2 mutations were significantly more likely to develop MDS/MPN that those with dominant SRSF2 followed by secondary TET2 mutations [OR 0.26 (0.12-0.62), $P=.0013$ vs. OR $0.84(0.38-2.24), P=0.7]$.

Focusing on other pairs of dominant and secondary mutations, 12 pairings affected risk characteristics (Fig. 3c; Supplementary Table 3). For example, dominant RUNX1 mutations followed by secondary ASXL1, STAG2, or IDH2 mutations were more likely associated with high-risk subtypes. Whereas, dominant SF3B1 mutations followed by secondary DNMT3A or JAK2 mutations were more likely associated with low-risk subtypes. Thus, distinct phenotypic features are due to specific dominant/secondary combinations, and multiple combinations can result in overlapping phenotypes. Of note is that secondary mutations in the 
a

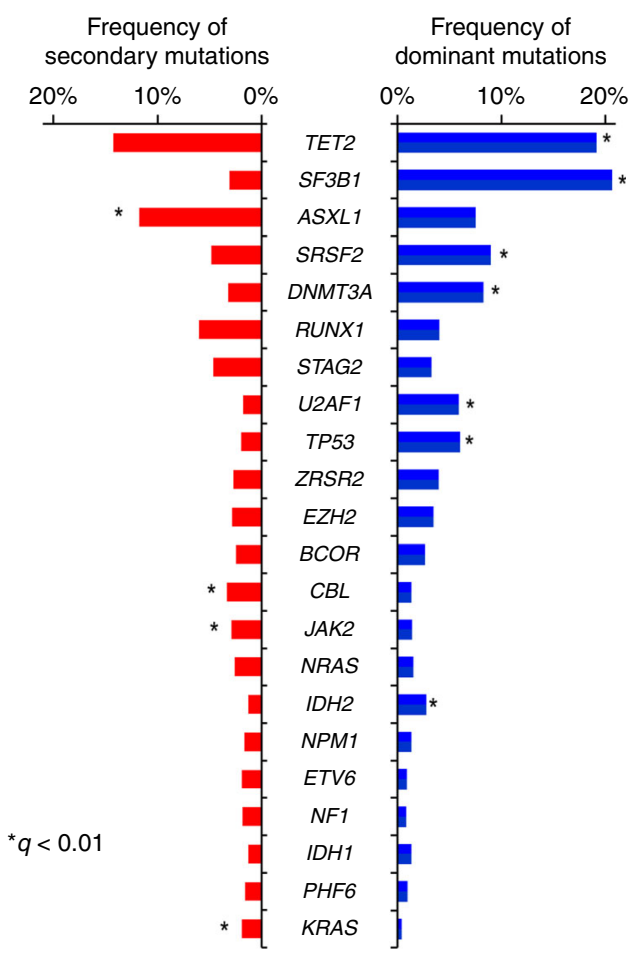

e

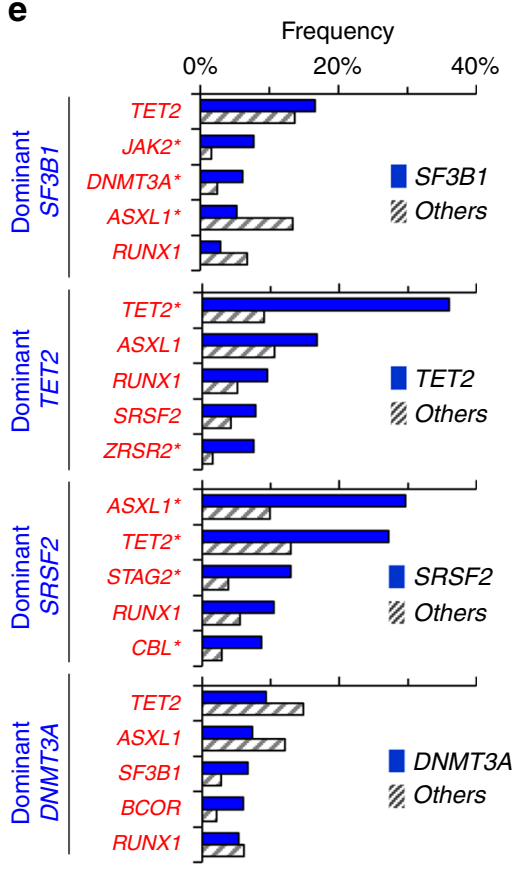

b

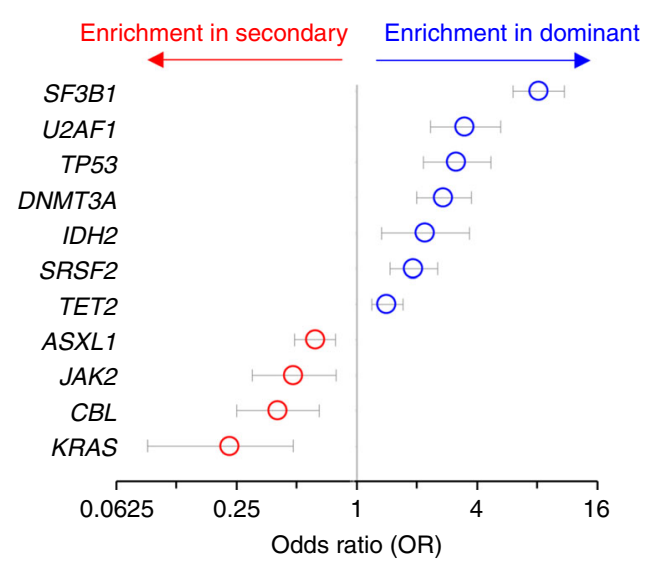

C

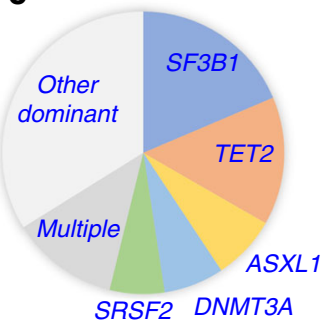

d

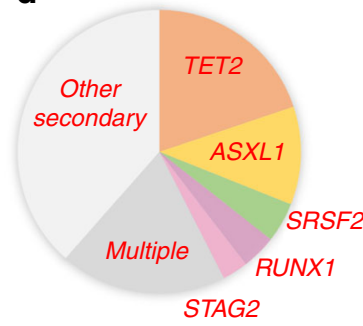

Secondary mutations
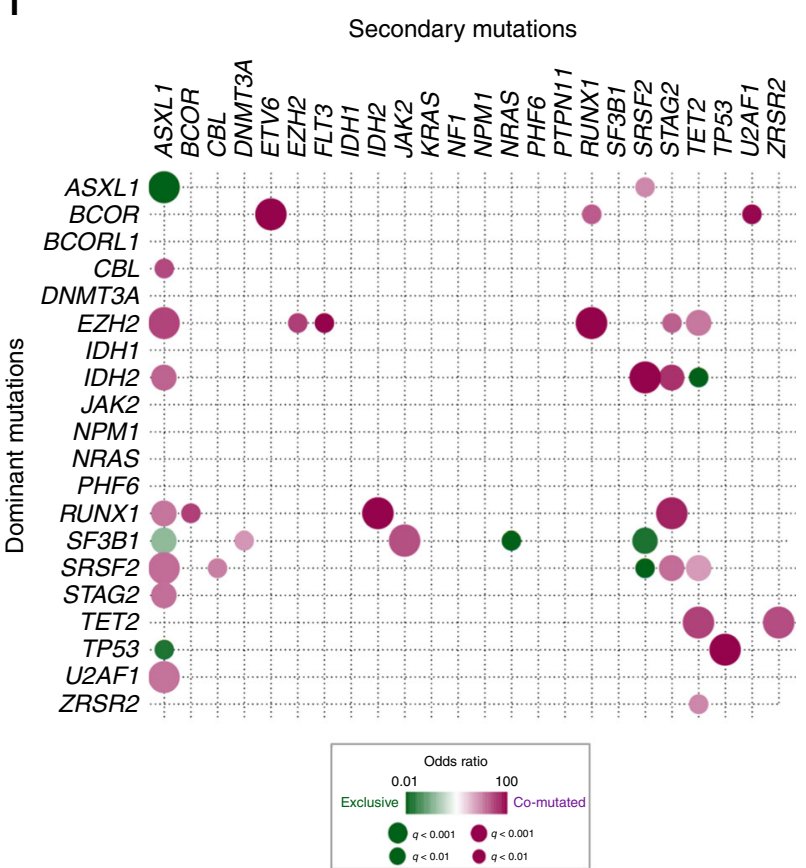

Fig. 2 Comparison of dominant- and secondary mutations, and their combinations. a Comparison of frequencies of dominant vs. secondary recurrent somatic mutations (recurrent is defined here as $>2 \%, n=22$ ); ${ }^{*}$ indicate $q<0.01$, where $q$ is the Benjamini-Hochberg corrected Fisher's exact test $P$-value. b Odds ratios and $95 \%$ confidential intervals of genes more likely to have dominant (blue) or secondary (red) mutations. c, $\mathbf{d}$ Proportion of top five frequent genes for (c) patients with dominant mutations, and (d) those with secondary mutations. Patients with multiple dominant or secondary mutations in the top five genes belong to the group called Multiple. e Nonrandom associations between dominant and secondary mutations. Shown are secondary mutations in each of top four dominant mutational groups. Bars indicate frequencies of secondary mutations in patients with (blue) vs. without (striped) dominant mutation. ${ }^{\star} q<0.01$, Fisher's exact test $P$-values with Benjamini-Hochberg correction. $\mathbf{f}$ Correlation of dominant and secondary mutations. Recurrent dominant $(>1 \%, n=16)$ and secondary mutations $(>1 \%, n=22)$ are given in the $y$ - and $x$-axes, respectively. Co-occurrence and mutually exclusivity are encoded in purple and green color gradients, respectively. Circle sizes encode $q$-values (Fisher's exact test $P$-values with Benjamini-Hochberg corrections). 
Table 2 Significantly correlated pairs between dominant and secondary events.

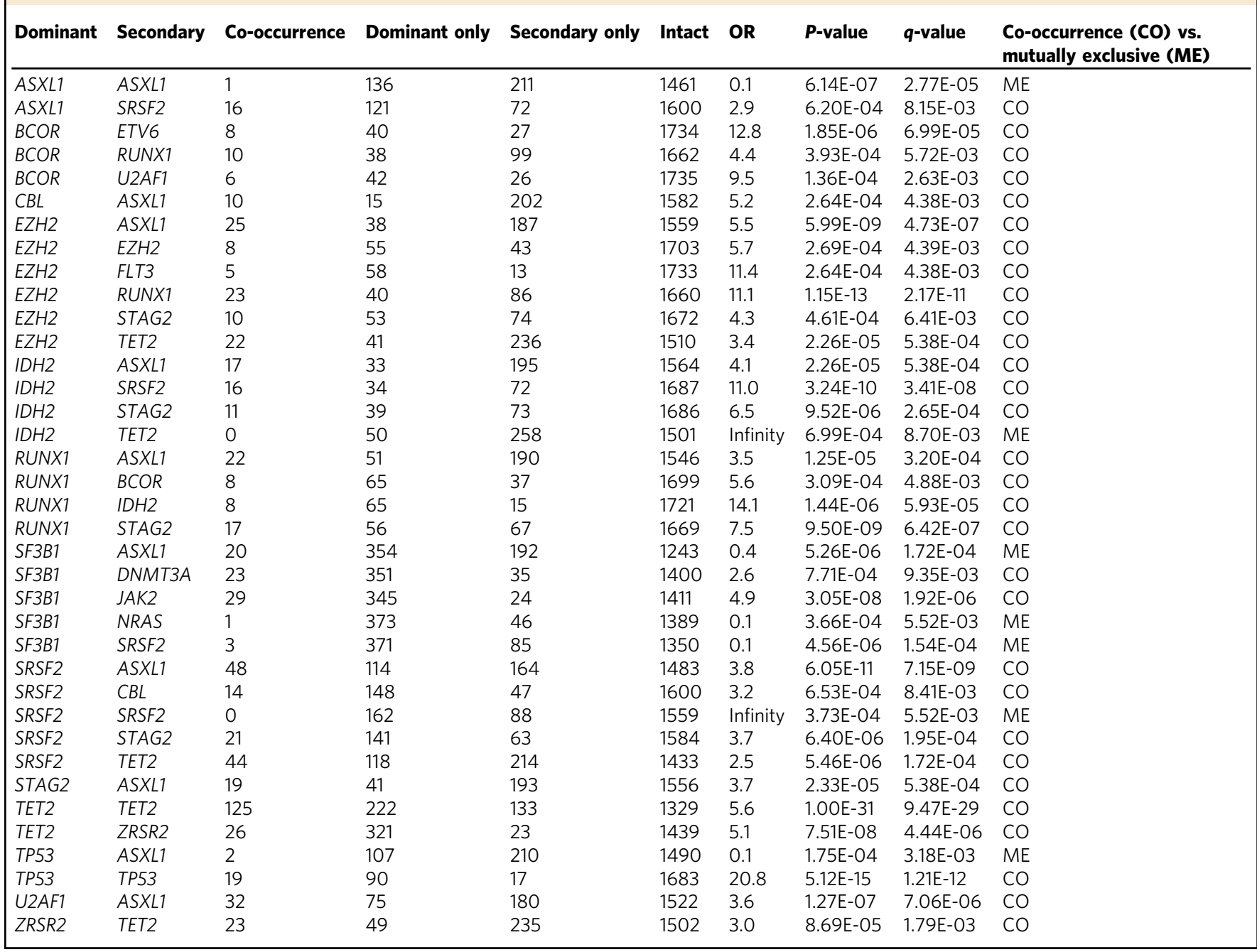

same gene modify the phenotypic features. For example, patients with bi-allelic EZH2 alterations were more likely associated with high-risk subtypes and MDS/MPN features compared monoallelic EZH2 (Supplementary Fig. 13).

Prior studies ${ }^{30,31}$ of MDS patients have demonstrated that the prognostic significance of TP53 mutations depends in part on their VAF, with smaller clones having a less adverse impact. Of the 126 patients with TP53 mutations in our cohort, 70 had a $\mathrm{VAF}>0.4$ and 56 had a VAF $=<0.4$. The TP53 mutant patients with a VAF $>0.4$ had significantly shorter overall survival than those with a $\mathrm{VAF}=<0.4$ [median OS 8 vs. 17.8 months, hazard ratio (HR) 1.93 (95\% CI: 1.23-3.04), $P=0.004$ ] (Supplementary Fig. 14a). In addition, TP53 mutant patients with a VAF $=<0.4$ had worse survival compared with TP53 wild-type (WT) patients [median OS 17.8 vs. 44 months, HR 1.95 (1.36-2.79), $P=$ 0.0003 ]. Focusing on clonal architecture, dominant and secondary TP53 mutations were identified in 109 and 36 patients, respectively. Fifteen percent (19/126) of the TP53 mutant patients had both a dominant and secondary mutation (Supplementary Fig. 14b). Patients with (i) both dominant and secondary, and (ii) only dominant TP53 mutations had significantly shorter overall survival than those WT for TP53 (median OS 7.33 vs 12.2 vs. 44 months, $P<0.0001$ ). HR for both group vs. WT was 4.31 (2.53-7.33), whereas, those with dominant or secondary TP53 mutations vs. WT were $2.92(2.23-3.82)$ or $1.78(0.98-3.23)$, respectively. In fact, 14 dominant/secondary mutations impacted survival (Supplementary Fig. 15). Focusing on 37 distinctive gene pairs, 9 co-mutations were associated with a poor prognosis (Fig. 4a; Supplementary Fig. 16). For instance, even though the frequent co-occurrence of a dominant EZH2 mutation and secondary ASXL1 or RUNX1 mutations, they were associated independently with poor outcome. Whereas dominant $B C O R$ and secondary $U 2 A F 1$ mutations did not affect survival, their cooccurrence was associated with shortened survival.

Associations with response to hypomethylating therapy. Focusing on specific treatments, we identified 179 patients who were treated with hypomethylating agents (HMAs). Patients with either complete response (CR), partial response, or hematologic improvement were considered responders. According to IWG response criteria for $\mathrm{MDS}^{32}$, 37\% (67/179) were responders, including $18 \%(33 / 179)$ achieving CR; 112 patients were nonresponders.

The presence of both TET2 and/or ASXL1 mutations was predictive of responsiveness (including CRs) or refractoriness to HMA therapy, respectively (Fig. 4b). For example, TET2 mutations were associated with higher response rates than WT [53\% (23/43) vs. $32 \%$ (44/136); OR $2.4(1.2-4.9), P=0.014]$, including CR, whereas, ASXL1 mutations were associated with lower response rates. Prior studies ${ }^{33}$ showed that TET2 mutant patients with WT for ASXL1 had a higher response rate to HMA 
a

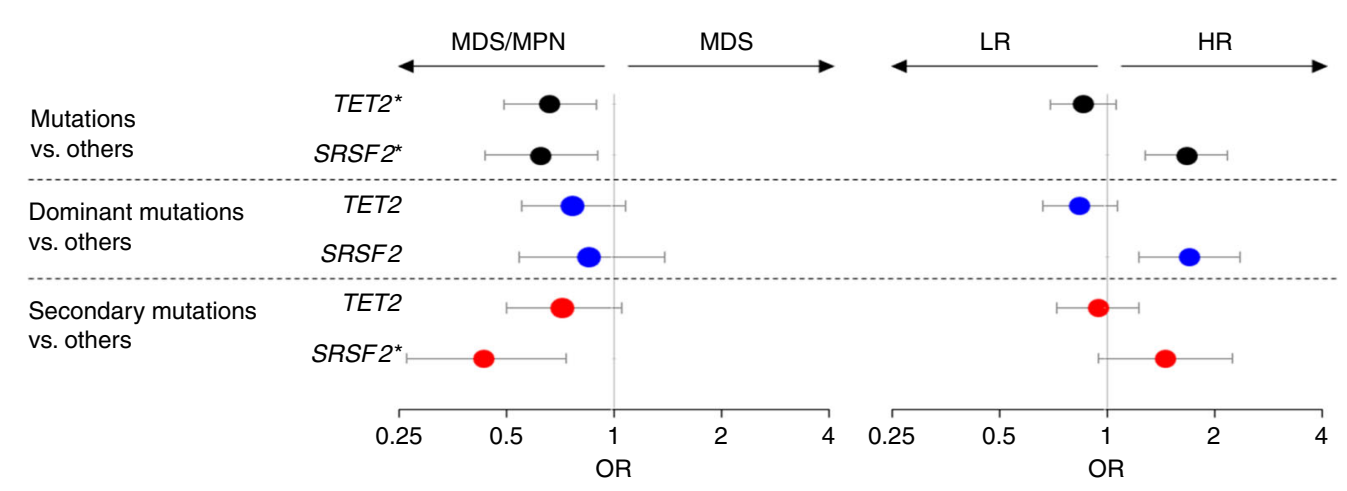

b
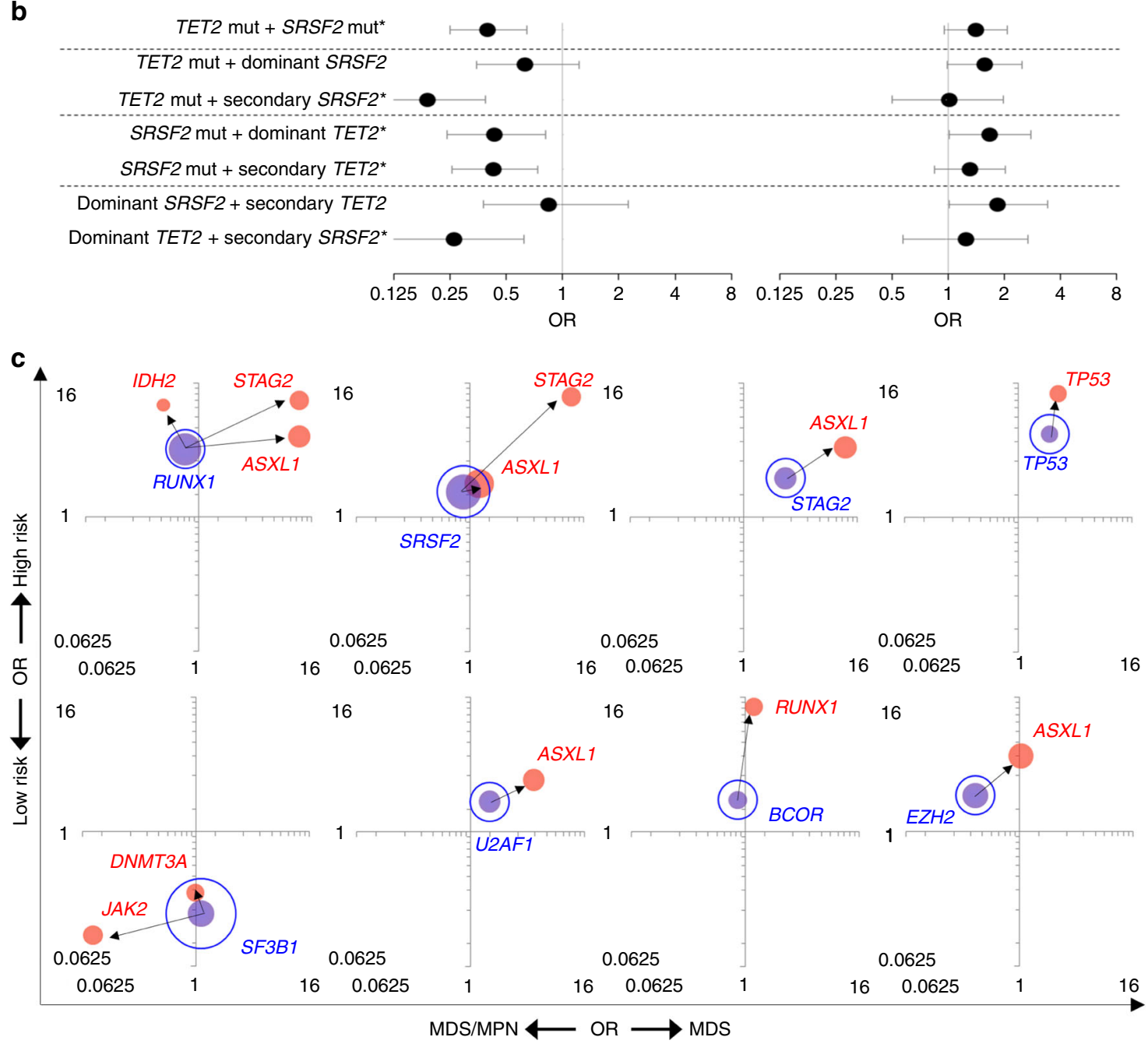

Fig. 3 Clinical effects for frequent pairs of dominant and secondary mutations. a, b Clinical features for patients with TET2 and/or SRSF2 mutations. Forest plot shows the odds ratios (OR) of MDS vs. MDS/MPN (left side) and high (HR) vs. low-risk (LR) subtypes (right side) for genetic events of (a) single mutation and (b) pairs of mutations, respectively. The significance was determined by Fisher's exact test; ${ }^{*} P<0.05$ (MDS vs. MDS/MPN). Circle and error bars indicate OR and 95\% confidence interval. Blue and red dots depict dominant and secondary mutations. c Clinical feature dependence on mutation combinations. Phenotypic features compared are MDS $(n=1583)$ vs. MDS/MPN $(n=226)$ on the $x$-axis, and low- $(n=1043)$ vs. high-risk $(n=766)$ groups on the $y$-axis. The area of each bubble indicates the frequency of mutations identified in 1809 MDS patients. Bubbles colored blue and red showed dominant and secondary mutations, respectively. Arrows indicate mutation sequence/ordering. All pairs of dominant and secondary mutations with significant phenotypic effects are highlighted by filled bubbles connected by arrows.

therapy, the results that were recapitulated in our cohort $[61 \%$ $(19 / 31)$ vs. $32 \%$ (48/148); OR 3.3 (1.5-7.5), $P=0.003]$. On the other hand, these significant associations disappeared in patients with both TET2 and ASXL1 mutations $(P=0.76)$. This suggests that ASXL1 mutations override the advantage of TET2 mutations.
In terms of clonal architecture, patients with dominant TET2 mutations demonstrated higher response rates [56 vs. $33 \%$; OR 2.6 (1.2-5.7), $P=0.017$ ]; however, secondary TET2 mutations did not $(P=0.16)$ (Fig. 4c). Although dominant or secondary ASXL1 mutations could not predict response, patients with secondary 
a
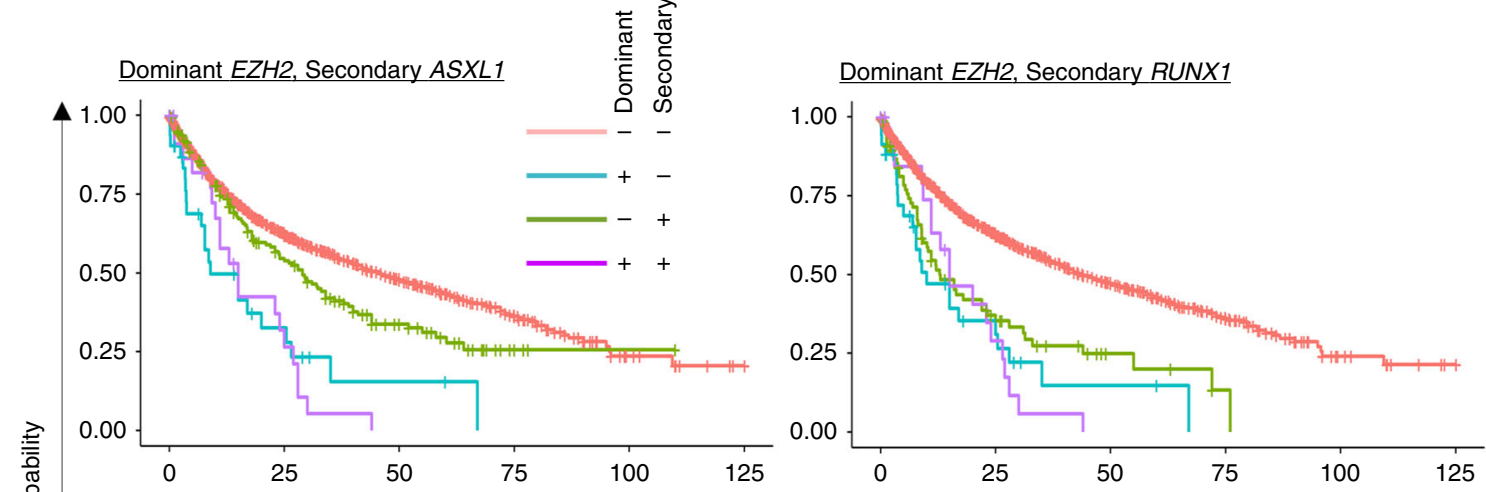

Dominant RUNX1, Secondary BCOR

Dominant BCOR, Secondary U2AF1
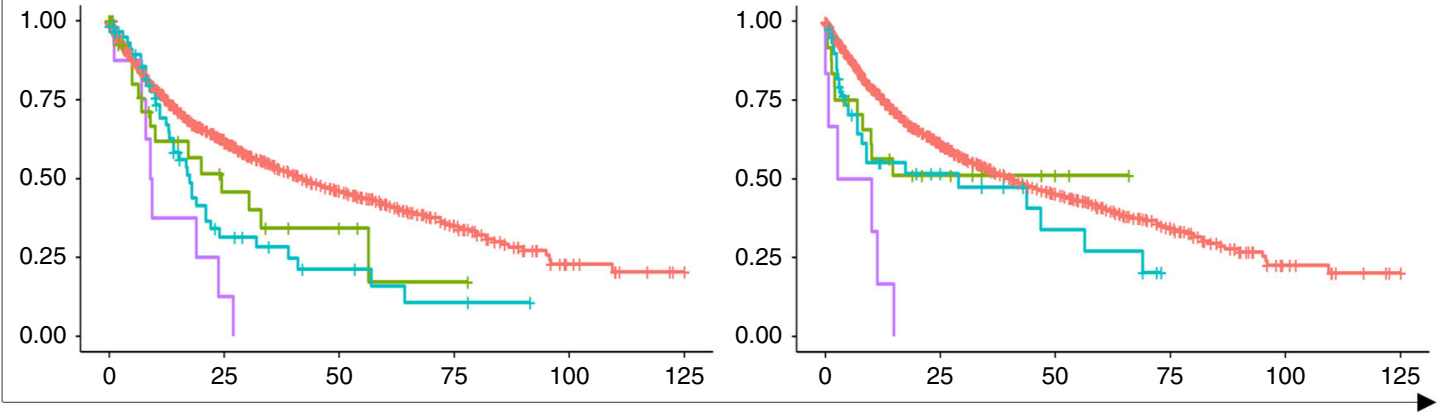

Months

b
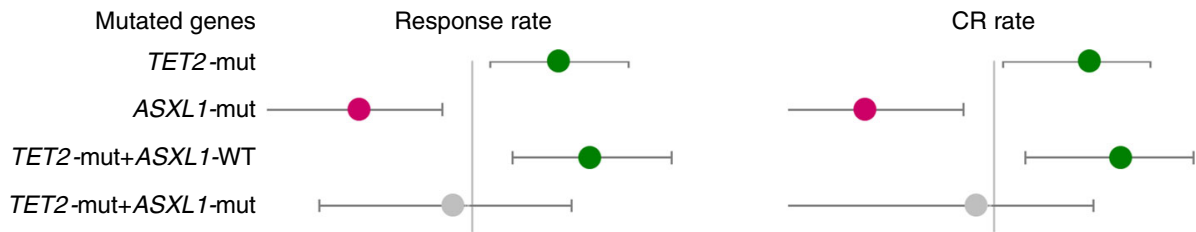

C
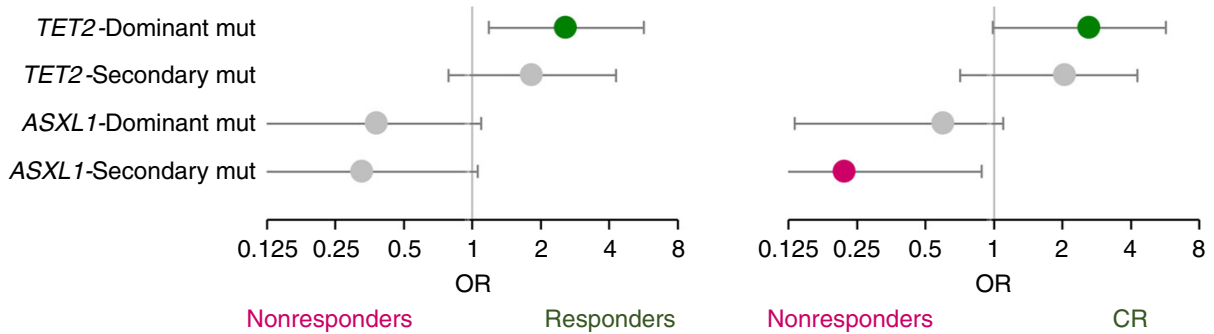

Nonresponders

CR

Fig. 4 Survival and response of hypomethylating therapy for dominant and secondary mutations. a Kaplan-Meier curves for pairs of dominant and secondary mutations with significant effect. Representative four co-occurring pairs are shown in above the curves. Each graph depicts four different group results (both dominant and secondary mutation, only dominant mutations, only secondary mutations, and neither dominant nor secondary mutations). b, c Associations between the presence of mutations and responses for hypometylkationg therapy. Circle and whiskers indicate odds ratios (OR) and 95\% confidence interval. Green circle depict responder (left panel) or complete response (CR) (right panel), whereas pink circle does nonresponders in both panels. Gray circle shows no significance. b Patients with TET2 or ASXL1 mutations. c Those with dominant or secondary mutations for TET2 or ASXL1 mutations.

ASXL1 mutations were statistically unlikely to achieve CR [0 (0/ $14)$ vs. $25 \%(33 / 131), P=0.04)]$. DNMT3A and TP53 mutations were not associated with response rates, while $U 2 A F 1$ mutations were associated with lower response rates $(P=0.047)$ (Supplementary Table 4).

Distinct features of $\mathrm{CH}$-derived and de novo MDS. Subclinical clonal expansions, referred to as clonal hematopoiesis $(\mathrm{CH})$, are present in the blood of otherwise healthy individuals. While many $\mathrm{CH}$-associated mutations occur in MDS, only a small proportion of asymptomatic individuals with $\mathrm{CH}$ progress to MDS. Because of the paucity of direct prospective data to project the fraction of $\mathrm{CH}$-derived cases based on the spectrum of dominant hits, we stipulate that (i) a proportion of $\mathrm{CH}$ mutations will eventually serve as ancestral hits that manifest as MDS upon acquisition of additional genetic alterations, and (ii) MDS from antecedent $\mathrm{CH}$ may be an MDS disease subtype that is distinct from de novo 
a

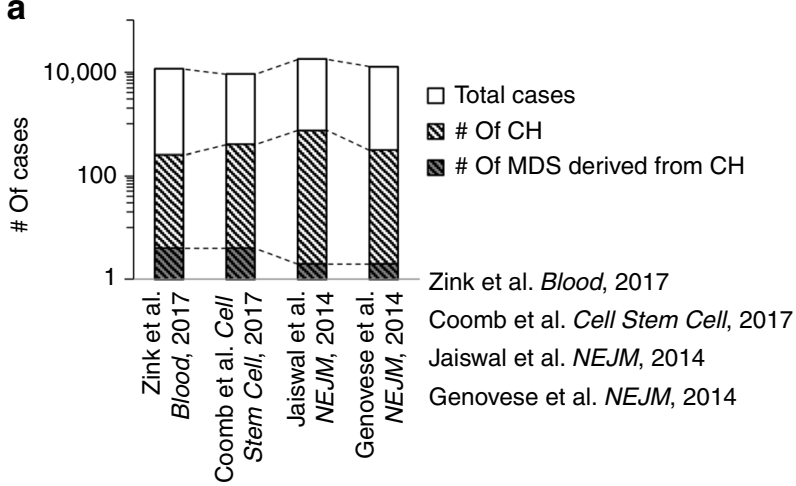

C

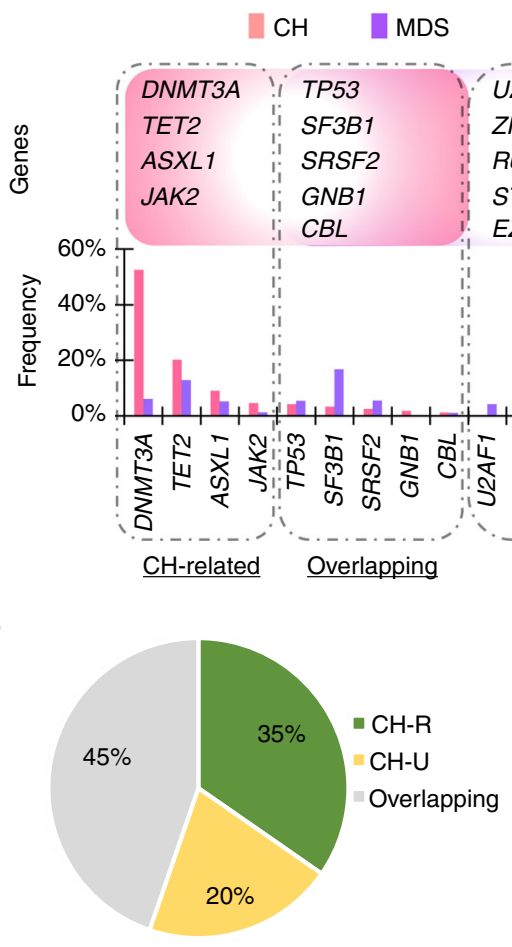

g

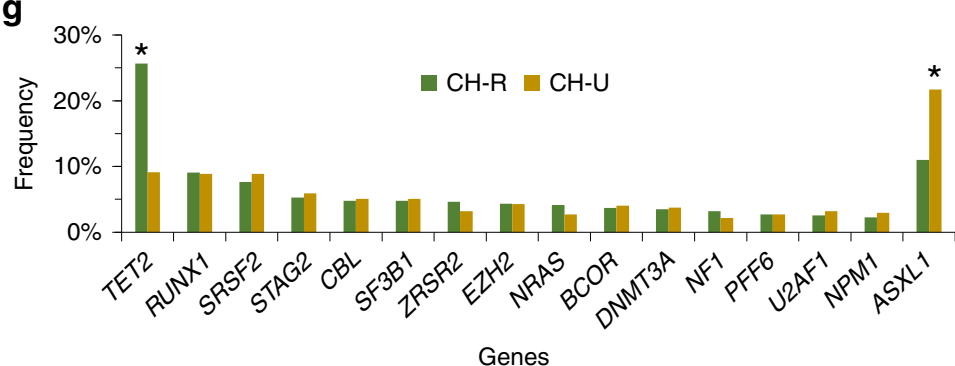

d

CH-unrelated

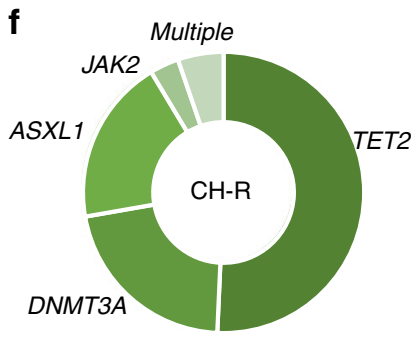

b

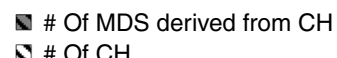

s \# Of $\mathrm{CH}$

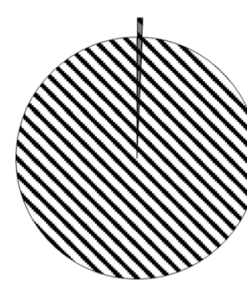

$\mathrm{CH}->\mathrm{MDS}$

$0.71 \%(12 / 1693)$
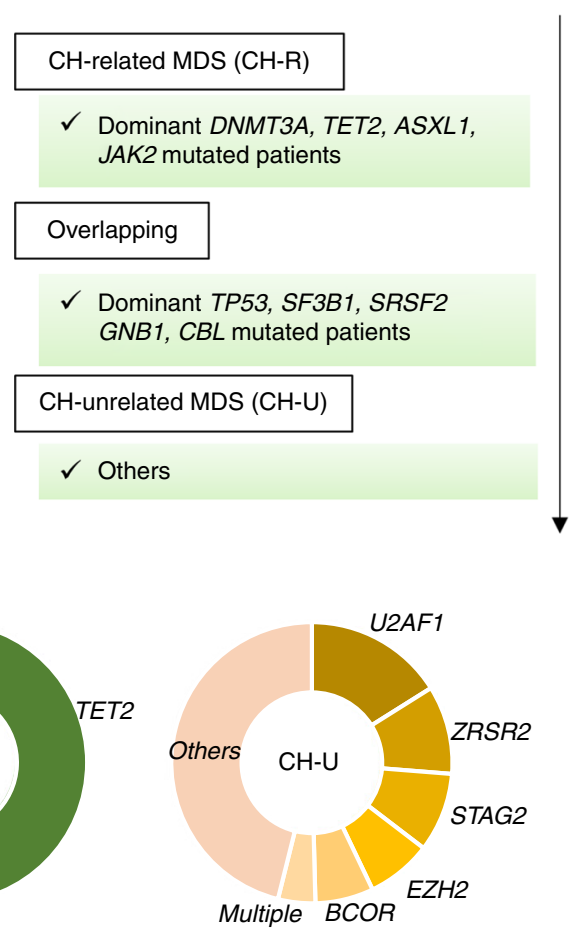

h

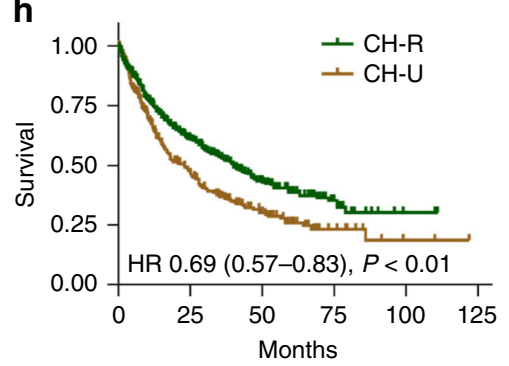

MDS, characterized by more penetrant primary hits. Separating ancestral/dominant vs. secondary hits in MDS patients and comparing their frequencies to those (obtained through metaanalysis) in $\mathrm{CH}$ may enable molecular and clinical characterization of CH-related MDS.

For our analysis, the targeted panel covered $94.6 \%$ of $\mathrm{CH}$ mutations discovered by whole-exome sequencing ${ }^{34}$ (Supplementary Fig. 17a). We have also sequenced additional patients for the four genes $(5.4 \%)$ which were omitted from our targeted panel genes, which resulted in only $<0.9 \%$ of patients being affected (Supplementary Fig. 17b). When we compared mutated genes in a historical cohort of 1693 healthy $\mathrm{CH}$ individuals including 12
MDS patients who derived from $\mathrm{CH}$ to dominant mutations in our MDS patients (Fig. 5a, b; Supplementary Figs. 18, 19 and Supplementary Table 5), mutations in DNMT3A, TET2, ASXL1, and $J A K 2$ were more frequent in $\mathrm{CH}$ than in MDS (e.g., DNMT3A; 52 vs. $8 \%, P<0.001$; Fig. 5c; Supplementary Table 6). Hence, MDS patients with dominant mutations in these four genes were defined as $\mathrm{CH}$-related MDS (CH-R; Fig. 5d). Other dominant mutations such as U2AF1, RUNX1, and STAG2, which were not identified in individuals with $\mathrm{CH}$ were deemed $\mathrm{CH}$ unrelated MDS (CH-U). In between, mutations in TP53, SF3B1, SRSF2, GNB1, and CBL were identified with similar frequencies in both cohorts (e.g., TP53; 4 vs. $5 \%, P=0.11$ ), and such cases will 
Fig. 5 Characteristics of clonal hematopoiesis (CH)-related MDS. a The number of cases with sequenced, $\mathrm{CH}$ and MDS derived from $\mathrm{CH}$ in different four cohorts. Four papers are also shown. b Penetrance from $\mathrm{CH}$ to $\mathrm{CH}$-derived MDS. Pie chart shows the number of cases with $\mathrm{CH}$ and $\mathrm{CH}$-derived $\mathrm{MDS}$ in merged four cohorts. c Genes and frequencies of mutations in $\mathrm{CH}$ and MDS cohorts. Frequencies of mutation in the $\mathrm{CH}$ cohort and of dominant mutations in the MDS cohort are shown light pink and purple bar graphs, respectively; four genes (DNMT3A, TET2, ASXL1, and JAK2) which were mutated in MDS and frequently in $\mathrm{CH}$ are shown on the left. Five genes (TP53, SF3B1, SRSF2, GNB1, and CBL) which were mutated with similar frequency in both cohorts are shown in the middle. Seven genes that are frequently dominant mutants in MDS and uncommon in $\mathrm{CH}$ are shown on the right. These distinct groups are marked $\mathrm{CH}$-related $(\mathrm{CH}-\mathrm{R})$, overlapping, and $\mathrm{CH}$-unrelated $(\mathrm{CH}-\mathrm{U})$. d Estimating $\mathrm{CH}$-related MDS. MDS patients with dominant mutations of DNMT3A, TET2, ASXL1, and JAK2 were defined as CH-related. Those with TP53, SF3B1, SRSF2, GNB1, and CBL were defined overlapping. Finally, those with dominant mutations were defined as $\mathrm{CH}$-unrelated. e Proportion of $\mathrm{CH}-\mathrm{R}, \mathrm{CH}-\mathrm{U}$ and Overlapping MDSs. Pie chart shows MDS patients $(n=1809)$ divided into $\mathrm{CH}-\mathrm{R}$ $(n=627), \mathrm{CH}-\mathrm{U}(n=373)$, and overlapping MDS $(n=809)$. f Fractions of genetic mutations in $\mathrm{CH}-\mathrm{R}$ and $\mathrm{CH}-\mathrm{U}$ MDSs. Pie charts show fractions of genes with dominant mutations in $\mathrm{CH}-\mathrm{R}(n=627)$ and $\mathrm{CH}-\mathrm{U}$ MDSs $(n=373)$. $\mathbf{g}$ Frequency of secondary mutations in $\mathrm{CH}-\mathrm{R}$ (green) and CH-U MDS (yellow). ${ }^{*} q<0.01$ (Fisher's exact test $P$-values with Benjamini-Hochberg correction). $\mathbf{h} \mathrm{CH}-\mathrm{R}$ MDS cases have a better prognosis. Kaplan-Meier curves for overall survival among patients with $\mathrm{CH}-\mathrm{R}$ MDS (green) and with $\mathrm{CH}-\mathrm{U}$ MDS (yellow) are depicted. Tick marks indicate censored data.

be denoted as overlapping MDS patients, whereby founder mutations lead to directly to MDS or indirectly though $\mathrm{CH}$.

We compared clinical, molecular, and demographic features of $\mathrm{CH}-\mathrm{R}$ vs. $\mathrm{CH}-\mathrm{U}$. There were 627 (35\%) $\mathrm{CH}-\mathrm{R}$ and 373 (20\%) $\mathrm{CH}-\mathrm{U}$ cases, out of 1809 MDS patients (Fig. 5e); $97 \%$ of patients with CH-R had at least one dominant TET2 (55\%), DNMT3A (24\%), or ASXL1 (22\%) mutation (Fig. 5f). The top five dominant mutations in patients with $\mathrm{CH}-\mathrm{U}$ were U2AF1 (19\%), ZRSR2 (12\%), STAG2 (10\%), EZH2 (9\%), and BCOR (8\%). In all, $54 \%$ of patients with $\mathrm{CH}-\mathrm{U}$ had at least one of these mutations. Focusing on secondary mutations, patients with $\mathrm{CH}-\mathrm{R}$ had a significantly higher frequency of secondary TET2 mutations than those with $\mathrm{CH}-\mathrm{U}$ (e.g., TET2, 26 vs. 9\%, $P<0.001$; Fig. 5g). In contrast, secondary ASXL1 mutations were more frequent in $\mathrm{CH}-\mathrm{U}$ [11 vs. $22 \%, P<0.001]$. $\mathrm{CH}-\mathrm{R}$ cases were older and had more low-risk subtypes and normal karyotype than $\mathrm{CH}-\mathrm{U}$ cases [e.g., average age 72 vs. 68 -years old, $P<0.001$; low-risk subtypes 58 vs. $44 \%, P$ $<0.001$ ] (Table 3 ). Patients with $\mathrm{CH}-\mathrm{R}$ also had a better prognosis than those with $\mathrm{CH}-\mathrm{U}$ [HR 0.69 (0.57-0.83), $P<0.001$; Fig. 5h].

\section{Discussion}

Our goal was to study the clonal architecture of MDS to clarify the impact of mutations and their combinations on clinical phenotypes, and to assess the role of $\mathrm{CH}$-associated mutations in frank MDS. For that purpose, we generated a well-annotated large cohort of untreated MDS patients that would allow us to account for the tremendous clinical heterogeneity and the corresponding genotypic diversity. Our initial results were concordant with previous studies with regard to the general mutational pattern ${ }^{35}$, validating the basis for subsequent analyses. In contrast to previous studies, we systematically explored correlations between mutational clonal hierarchy and clinical phenotypes.

After inferring clonal architecture by the stringent bioanalytic pipeline PyClone, which has been validated by single-cell sequencing, recurrently mutated genes could thus be characterized by the proportion with which they were dominant vs. secondary, i.e., initiating or supporting the clone; some genetic mutations are predominantly initiating, but with a propensity to occur at various stages of the clonal hierarchy. For instance, TP53 or SF3B1 mutations were more likely to be dominant, and ASXL1 and KRAS mutations were more like to be secondary. Dominant mutations defined distinct subgroups within which multiple secondary mutations accumulated. Among these pairings, we cataloged the most common combinations and characterized the extent to which they affected morphological and clinical phenotypes. In total, consequential 38 significant dominant/secondary pairs were identified. In addition to confirmatory relationships, such as dominant SF3B1 and secondary JAK2 mutations in RARS-T ${ }^{36,37}$, our analysis yielded several new associations.

\begin{tabular}{|c|c|c|c|c|}
\hline & & $\begin{array}{l}\text { CH-R } \\
(n=627)\end{array}$ & $\begin{array}{l}\text { CH-U } \\
(n=373)\end{array}$ & P-value \\
\hline \multirow[t]{2}{*}{ Age } & Mean & 72.4 & 67.5 & $<0.0001$ \\
\hline & $>60$ y $(\%)$ & $564(92 \%)$ & $283(77 \%)$ & $2.4 \times 10^{-10}$ \\
\hline Sex & Male (\%) & $400(63 \%)$ & $254(68 \%)$ & 0.18 \\
\hline \multirow[t]{4}{*}{ Diagnosis } & & & & $2.0 \times 10^{-6}$ \\
\hline & MDS & $499(80 \%)$ & $264(71 \%)$ & 0.002 \\
\hline & MDS/MPN & $86(14 \%)$ & $46(12 \%)$ & 0.56 \\
\hline & sAML & $42(7 \%)$ & $63(17 \%)$ & $6.4 \times 10^{-7}$ \\
\hline \multirow[t]{3}{*}{ Risk classification } & & & & $4.7 \times 10^{-5}$ \\
\hline & Low risk & $362(58 \%)$ & 165 (44\%) & \\
\hline & High risk & $265(42 \%)$ & $208(56 \%)$ & \\
\hline \multirow[t]{5}{*}{ Karyotypes } & & & & \\
\hline & Normal & $389(62 \%)$ & $186(50 \%)$ & 0.0002 \\
\hline & Complex & $43(7 \%)$ & $33(9 \%)$ & 0.27 \\
\hline & $-5 / \operatorname{del}(5 q)$ & $59(9 \%)$ & $38(10 \%)$ & 0.74 \\
\hline & $-7 / \operatorname{del}(7 q)$ & $44(7 \%)$ & $41(11 \%)$ & 0.034 \\
\hline \multirow{9}{*}{$\begin{array}{l}\text { Number of } \\
\text { mutations } \\
\text { Other mutations }{ }^{a}\end{array}$} & Mean & 3.2 & 2.6 & $<0.0001$ \\
\hline & & & & \\
\hline & RUNX1 & $76(12 \%)$ & $60(16 \%)$ & 0.078 \\
\hline & $E Z H 2$ & $53(8 \%)$ & $45(12 \%)$ & 0.064 \\
\hline & ZRSR2 & $53(8 \%)$ & $55(15 \%)$ & 0.0022 \\
\hline & STAG2 & $47(7 \%)$ & $60(16 \%)$ & $3.2 \times 10^{-5}$ \\
\hline & U2AF1 & $46(7 \%)$ & $81(22 \%)$ & $2.5 \times 10^{-10}$ \\
\hline & $B C O R / L 1$ & $39(6 \%)$ & $60(16 \%)$ & $1.0 \times 10^{-6}$ \\
\hline & IDH1/2 & $37(6 \%)$ & $53(14 \%)$ & $1.0 \times 10^{-5}$ \\
\hline
\end{tabular}

aFrequently mutated genes, except for the nine genes identified in CHIP

The concept of dominant or founder lesions (TET2-, SRSF2-, or TP53-initiated disease etc.) would simplify molecular classification of MDS types. Specific dominant and secondary mutations, and their combinations significantly affected dysplasia (MDS vs. MDS/MPN), progression (higher- vs. lower-risk subtypes), survival and response of HMA therapy. For instance, TET2 mutations were neutral with regard to the morphology, but they invited secondary hits such as SRSF2, resulting in subsequent drift toward an MDS/MPN phenotype and high-risk diseases. Similarly, dominant EZH2 and secondary ASXL1 or RUNX1 mutations were associated with high-risk subtypes and cooperatively led to a poor prognosis. Moreover, analysis of the rank of TP53 mutation within clonal structure is instructive: i.e., patients with dominant mutations, which reflects high clonal burdens, had worse prognosis compared with those with secondary mutations; those with both had the worst prognosis. This is consistent with bi-allelic TP53 hits conveying typically the highest risk, as 
previously reported ${ }^{30,31}$. Finally, distinction of dominant hits can be also predictive of therapy responses, e.g., dominant TET2 or secondary ASXL1 mutations were more associated with response rate to HMA therapy than secondary TET2 or dominant ASXL1 mutations. Thus, dividing genetic mutations into dominant vs. secondary types may help us understand MDS pathogenesis and increase their predictive value as biomarker. Our results provide a catalog of dominant and secondary mutations and the phenotypic drives they exert and also how the impact of secondary hits depends on the ancestral hits preceding them. Irrespective of the dominant mutations, most secondary mutations increased the odds of advanced disease.

Obviously, resolution of identifying dominant and secondary hits solely by sequencing is a limitation of our study. Moreover, assessment of mutations at a single time point are not capable of definitive determination whether secondary mutations are present within the same subclone. Recent technologies enable singlecell analysis 2,38 and genotyping of hematopoietic progenitor colonies of hematopoietic stem cells in MDS ${ }^{27}$. These methods are ideal for confirming clonal substructure, and tracking the clonal evolution of $\mathrm{MDS}^{39}$. While these technologies analytically are superior in individual patients ${ }^{40}$, given the time and cost investment, the PyClone approach is more practical clinically, and allows more generalizable results in large cohorts representative of molecular and clinical diversity of MDS.

Sequencing bone marrow DNA in patients with $\mathrm{CH}$ who later developed AML demonstrated that the leukemia was clonally derived from the previously identified $\mathrm{CH}^{34}$. Hence, acquired genetic mutations at the $\mathrm{CH}$ phase could represent early hits in MDS. In lieu of the very few prospective cases studied serially from $\mathrm{CH}$ to the evolution of MDS in the literature, we stipulated that mutations with a frequency lower in MDS than in $\mathrm{CH}$ point toward $\mathrm{CH}$ derivation, while those with present only in MDS (and absent in $\mathrm{CH}$ ) evolve without prodromal $\mathrm{CH}$ phase. Accordingly, based on dominant hits in $\mathrm{CH}^{18,19}$ and MDS, we determined which patients should be classified as $\mathrm{CH}-\mathrm{R}$ MDS or de novo MDS (CH-U MDS) and evaluated molecular and clinical differences between these two categories. Several reports of $\mathrm{CH}$ mutations shared with MDS yielded approximations of the fraction of mutations that were $\mathrm{CH}$-derived. The majority of $\mathrm{CH}$ related MDS had at least one dominant mutation of DNMT3A, TET2, and ASXL1, frequent secondary TET2 and ZRSR2 hits, older age, and better prognosis. Consistently, DNMT3A, TET2, and ASXL1 mutations, which are most commonly associated with age-related clonal hematopoiesis, during complete remission phase did not increase risk of relapse in $\mathrm{AML}^{41}$, whereas other mutations such as U2AF1 and RUNX1 (absent in $\mathrm{CH}$ ) could associate with relapse and lower rates of relapse-free survival ${ }^{6,42}$. These and other $\mathrm{CH}$-unrelated ancestral hits impart faster growth/progression to symptomatic MDS, and this may have precluded their appearance in healthy elderly ${ }^{43}$. In contrast, $\mathrm{CH}$ mutations do not appear to be drivers and gain this property with time during aging or upon acquisition of secondary hits. In terms of epidemiologic considerations, CH-R MDS patients in longitudinal studies ${ }^{18,22,44}$ showed mutational profiles that are distinct from $\mathrm{CH}-\mathrm{U}$ MDS.

Our targeted panel is lacking a few known recurrent genes (e.g., PPM1D, MYD88, ATM, and STAT3) with CH mutations, compared with the $\mathrm{WES}^{34}$. Therefore, a proportion of $\mathrm{CH}-\mathrm{U}$ MDS cases might have preceded $\mathrm{CH}$. To address this question, we searched for these mutations in an additional cohort, isolating them in $<0.9 \%$ of patients. It suggests that the initial omission of these genes might not result in major misclassification. Indeed, $P P M 1 D$, while frequent in $\mathrm{CH}$, was reported to be exceedingly rare in primary MDS and mostly present in therapy-related MDS/ $\mathrm{AML}^{45,46}$. Consistent with these findings, the two patients with
PPM1D mutations in our additional cohort had a previous history of chemotherapy or radiation therapy. Given that 1809 patients in our cohort had no history of chemotherapy or radiation, we anticipate $P P M 1 D$ mutations to be extremely rare.

Another limitation of our $\mathrm{CH}$ vs. MDS comparison is that no confirmatory samples were analyzed before the time point of MDS diagnosis. Prospective analyses that include sequencing of serial samples from identification of $\mathrm{CH}$ to development of MDS in many patients would be optimal for revealing the landscape of cases with $\mathrm{CH}$-derived MDS in greater detail. However, it might be difficult because of requirement large number of healthy donors and a long following-up time. We believe our hypothesis regarding the clinical and molecular characteristics of $\mathrm{CH}$-derived MDS represent an alternative method useful in various clinical applications.

In conclusion, a stringent bioanalytic pipeline called PyClone estimated the clonal architecture in MDS patients and these results can be used to assign each patient to dominant events, and thus to subgroups sharing the event. Our results show that in the process of clonal evolution, various initial hits are preferentially followed by a specific spectrum of secondary hits that shapes phenotypic and biologic features of MDS. Focusing on ancestral/ dominant events is instructive, it allows discrimination between MDS with antecedent $\mathrm{CH}$ and de novo MDS. Ancestral hits are the origins of MDS that determine unique characteristics in $\mathrm{CH}-\mathrm{R}$ vs. $\mathrm{CH}-\mathrm{U}$ MDS. Given the clinical characteristics of $\mathrm{CH}-\mathrm{R}$ MDS, detecting and monitoring $\mathrm{CH}$ may be warranted, particularly as biomarkers better predict risks and as preventive methods are developed and become more readily available.

\section{Methods}

Patients. A total of 1809 patients with MDS $(n=1446), \operatorname{MDS} / \mathrm{MPN}(n=212)$, and secondary AML (sAML) from MDS or MDS/MPN $(n=151)$ were screened and enrolled in this study (Table 1). Therapy-related MDSs were not included. In this paper 1169 patients (52\%) were previously reported in the paper by Makishima et al., which included 2250 patients (Supplementary Fig. 1). Additional data such as copy-number alterations and read counts of mutations were needed for inferring accurate clonal structures by PyClone, therefore only half of the patients could be used. To increase statistical power, we added another 640 patients which were uniformly annotated to contain all elements required. In addition, patients were selected who had fully annotated outcomes with follow up and had pathomorphological evaluation available. All samples were obtained after written informed consent, according to protocols approved by the Institutional Review Board of participating institutions (Cleveland Clinic's Institutional Review Board (IRB) and IRB-5024). In total, 212 patients were diagnosed with MDS/MPN; 121 patients (57\%) with CMML, 47 patients (22\%) with MDS/MPN-U, and 43 patients $(21 \%)$ with RARS-T. All sAML cases were determined derived from MDS $(n=137)$ or MDS/MPM $(n=14)$ by WHO classification to dichotomize morphologic features ${ }^{4}$. All cases are separated into two groups based on IPSS-R scores (low risk as $<=3.5$ and high risk as $>3.5$ ) according to the transformation risk ${ }^{47}, 58 \%$ of the patients (1043/1809) were low risk, whereas, 42\% (766/1809) were high risk (Table 1). GL DNA was obtained from either buccal mucosa or CD3-positive T cells, which were purified from peripheral blood with or without prior culture in the presence of PHA and IL- $2^{8}$. Tumor DNA was extracted from bone marrow or peripheral blood.

Whole-exome sequencing. WES was performed as previously described ${ }^{8,29}$. Paired disease and normal GL DNA were used. Whole-exome capture was accomplished through hybridization of sonicated genomic DNA to a bait cDNA library synthesized on magnetic beads (SureSelect Human All Exon $50 \mathrm{Mb}$ or V4 kit, Agilent Technologies). Captured targets were subjected to massively parallel sequencing using a HiSeq 2000 (Illumina) according to the standard protocol for 100-bp paired-end reads. Briefly, sequencing reads were aligned to the human genome (hg19) by a Burrows-Wheeler aligner (http://bio-bwa.sourceforge.net/). We used a GATK pipeline to extract candidate variants/polymorphisms and to remove sequencing errors. Validations were performed by Sanger or PCR amplicon sequencing as previously described ${ }^{29}$.

Targeted sequencing. Targeted sequencing was performed using a TruSeq Custom Amplicon (Illumina) or a custom cRNA bait library (SureSelect; Agilent Technology) as previously described $29,37,48$. The common 36 genes were overlapped in both targeted regions (Supplementary Table 1). Sequencing libraries were generated according to an Illumina paired-end library protocol. The enriched 
targets were subjected to massive sequencing using Hiseq 2000 or Miseq sequencer (Illumina), with sufficient read coverage (Supplementary Fig. 2). Variants were annotated using Annovar ${ }^{49}$ and filtered by removing: (i) synonymous singlenucleotide variants; (ii) variants only present in unidirectional reads; (iii) variants in repetitive genomic regions (Supplementary Fig. 3). Only variants with minimum depth of 20 and with 5 positive, high-quality reads, were considered. A bioanalytic pipeline, devised in-house, as previously described ${ }^{48}$, was applied to identify somatic mutations by comparison with sequenced controls and mutational databases, such as dbSNP138 $8^{50}, 1000$ Genomes $^{51}$ or ESP 6500 database, and Exome Aggregation Consortium (ExAC) ${ }^{52}$. Mapping errors were removed by visual inspection with the Integral Genomics Viewer. Validation by Sanger sequencing or PCR amplicon sequencing was performed as previously described ${ }^{48}$. Variant allelic frequencies were adjusted according to zygosity and copy number, based on conventional metaphase karyotyping/single-nucleotide polymorphism array results ${ }^{48}$. An overall accuracy of our platform for detection of somatic mutations was estimated to be $98.7 \%$ (74/75) (Supplementary Fig. 4).

\section{Copy-number analysis. We employed three different platforms (Karyotyping,} microarray, and digital copy-number analysis) for chromosomal alterations (Supplementary Fig. 5). In all, 95.7\% (1782/1809) patients were evaluated copy-number alterations, of which 1014 patients were studied by all three platforms. One is the conventional metaphase karyotyping, chromosome preparations were G-banded using trypsin Giemsa, and karyotypes were described in 1759 patients according to the International System for Human Cytogenetic Nomenclature ${ }^{53}$. Second is singlenucleotide polymorphism (SNP) array karyotyping for confirming metaphase cytogenetics and detecting copy-number normal loss of heterozygosity was performed as previously described ${ }^{10,54}$. Briefly, Affymetrix $250 \mathrm{~K}$ and 6.0 SNP arrays were used to evaluate copy number and loss of heterozygosity. Using our internal and a publicly available database (http://dgv.tcag.ca/dgv/app/home), the screening algorithm validated each lesion as somatic. Non-somatic lesions were excluded from further analysis. Affected genomic positions in each lesion were visualized and extracted using CNAG (v3.0) or Genotyping Console (Affymetrix) ${ }^{55,56}$. Third is digital copy-number analysis. Copy numbers for target exons were calculated, Let $d_{j}^{i, s}$ be the sequencing depth at the $i$ th nucleotide of the $j$ th exon in sample $s$, and the standardized depth of the $j$ th exon is calculated as $D_{j}^{s}=k_{s} \sum_{i} d_{i}^{j, s}$, where $k_{s}$ is determined so as to satisfy $k_{0}=\sum_{j} D_{j}^{s}$ for a fixed constant $k_{0}$ (=1, for example). Correlation co-efficient $\left(R=R^{s, t}\right.$ between two vectors $D_{i}^{s}$ and $D_{i}^{t}$ was calculated between sample $s$ and each of the 443 samples with completely normal copy numbers in aCGH $(t=1,2,3, \ldots, 443)$, respectively, through which the best $m_{0}$ correlations were identified between samples $T_{m}\left(m=1,2,3, \ldots, m_{0}\right)$. The copy numbers of the $i$ th target exon of sample s $\left(C n_{i}^{s}\right)$ was calculated as $C n_{i}^{s}=D_{i}^{s} / \hat{D}_{i}^{s}$, where $\hat{D}_{i}^{s}$ was provided by averaging $m_{0}$ samples, $\hat{D}_{i}^{s}=\sum_{m=1}^{m_{0}} D_{i}^{t_{m}} / m_{0}$ with $m_{0}=12$. Copy numbers were calculated for those exons, showing mean depth $>500$. Circular binary segmentation was used to identify discrete copy-number segments, using DNACopy by which segmented copy number $\left(\widehat{C n}_{i}^{s}\right)$ was defined for the $i$ th exon of sample s. Distribution of $\widehat{C n_{i}^{s}}$ was calculated for all samples, and those exons showing $\left|\widehat{C n_{i}^{s}}-E\left(\widehat{C n_{i}^{s}}\right)\right|>4 S D$ were thought to have copy-number losses or gains, respectively.

Estimation of tumor cell fraction. The estimated tumor cell fraction harboring the relevant mutation (TCFmut) was calculated from total copy number (TCN) of the region, minor allele-specific copy number $(\mathrm{AsCN})$, and observed variant allele frequency (VAFobs) as follows; TCFmut $=\mathrm{TCN} \times$ VAFobs (for deletions), TCFmut $=2$ VAFobs- $1+$ AsCN $[$ for UPD when VAF $>0.5(1-\mathrm{AsCN})(\mathrm{UPD}$ occurred later)], TCFmut $=2 \mathrm{VAFobs}[$ for UPD when $\mathrm{VAF}<0.5(1-\mathrm{AsCN})$ (mutation occurred later)] TCFmut $=2$ VAFobs (for regions without copy-number changes or UPD). For gains, since we cannot accurately estimate the correct TCN and tumor cell fractions, we tentatively assumed TCN as three, and accordingly tumor cell fractions can be estimated as TCFmut $=\mathrm{TCN} \times \mathrm{VAFobs}$.

Clonal structure inference. To detect subpopulations, clustering analysis of mutations was performed according to the Beta Binomial emission model implemented in PyClone ${ }^{28,57}$. Mutations in repetitive regions or indels, for which VAFs were poorly estimated, were not used for the analysis. Clustering was performed using a dynamic Tree Cut procedure. Density was used to model read counts (pyclone_beta_binomial), and PyClone ver 0.13 .0 was run with 10,000 iterations and a burn- in of 1000 with default setting 29,58 . A configuration file was generated for each patient by using the total copy-number option. The remaining parameters were set as follows: base measure parameters: alpha $=1$, beta $=1$; concentration value $=1$; prior shape $=1.0$, rate $=0.001$; for each sample, the error rate parameter was set to 0.001 . Clonal hierarchy was reduced to a one-dimensional ordered 2level feature space of primary/dominant, and secondary mutations (Fig. 1d). Mutations belonging to the largest clone (Pyclone cluster 1) were defined as dominant mutations and other clones as secondary mutations (Fig. 1e; Supplementary Fig. 7 and Supplementary Data 2).
Correlations of mutations. Frequent mutations and copy-number alterations $(>2 \%, n=30)$ were assessed for mutual correlation. Any combination of these variants was exhaustively tested in a pairwise manner using Fisher's exact test, and multiple testing was corrected with the Benjamini-Hochberg $q$-value (assumed significant when $q$-value $<0.01$ for coexistence and $<0.25$ for exclusion). Significant correlations were plotted with transition colors (magenta for positive and green for negative correlations), together with circle diameters indicating the degree of significance.

Association morphologic and features with mutations. The 1809 patients were dichotomized by morphologic features (MDS $(n=1583)$ vs. MDS/MPN $(n=226))$ and by progression to AML (low risk $(n=1043)$ vs. High risk $(n=766))$ by the criteria as shown above in patients. The bubble plot shows both odds ratios on the $x$ - and $y$-axes, respectively. Odds ratios for specific combinations of dominant and secondary mutations were calculated using the following steps; (i) count numbers of patients with (A) both dominant and secondary mutations, and (B) neither dominant nor secondary mutations; (ii) $2 \times 2$ (A) vs. (B) tables were dichotomized by morphology and progression to AML; (iii) odds ratios were then estimated. Combinations with significance in either morphology or progression to AML are shown (Fig. 3b, c; Supplementary Table 3).

CH-related MDS detection. Meta-analysis of mutation distribution within various large $\mathrm{CH}$ cohorts ${ }^{19,34,59-61}$ showed that the top eight most mutated genes account for nearly all of $\mathrm{CH}$ (Supplementary Figs. 17, 18). We then hypothesized that if a fraction of MDS originates from $\mathrm{CH}$, the compositions of dominant mutation should be similar. DNMT3A, TET2, ASXL1, and JAK2 mutations were significantly higher in $\mathrm{CH}$ compared with our MDS cohorts, hence MDS patients with dominant mutations of these four genes were defined as CH-related MDS (Fig. $5 c$, d). Mutations of the remaining five genes (SF3B1, SRSF2, TP53, GNB1, and CBL) were identified in both cohorts, so MDS patients with them were Overlapping, whereas those with other dominant mutations such as U2AF1, RUNX1, and STAG2 were $\mathrm{CH}$-unrelated MDS. Those without any mutations were overlapping because they could not be separated by these criteria.

Statistical analysis. Comparisons of proportions were performed by using twosided Fisher's exact tests. Paired data were analyzed by the Wilcoxon signed-rank test. Continuous variables were compared using the Mann-Whitney $U$ test. Kaplan-Meier methods were used to plot survival. The log-rank test was used to compare survival curves. Univariate and multivariate Cox proportional hazards survival analyses were also conducted. Analyses were performed with R (https:// www.r-project.org), SPSS software (IBM), and Prism (GraphPad). Significance was determined at a two-sided a level of 0.05 , except for $P$-values in multiple comparisons, which were adjusted according to the method described by Benjamini and Hochberg ${ }^{62}$.

Reporting summary. Further information on research design is available in the Nature Research Reporting Summary linked to this article.

\section{Data availability}

Genome data that support the findings of this study have been deposited through the database of Genotypes and Phenotypes (dbGaP) in the NCBI under accession number phs001898.v1.p1. All other remaining data are available within the Article and Supplementary Files, or available from the authors upon request.

Received: 14 January 2019; Accepted: 16 September 2019; Published online: 26 November 2019

\section{References}

1. Jacoby, M. A. et al. Subclones dominate at MDS progression following allogeneic hematopoietic cell transplant. JCI Insight 3, 98962 (2018).

2. Chen, J. et al. Myelodysplastic syndrome progression to acute myeloid leukemia at the stem cell level. Nat. Med. 25, 103-110 (2019).

3. Nagata, Y. \& Maciejewski, J. P. The functional mechanisms of mutations in myelodysplastic syndrome. Leukemia 1-16 (2019).

4. Arber, D. A. et al. The 2016 revision to the World Health Organization classification of myeloid neoplasms and acute leukemia. Blood 127, 2391-2405 (2016).

5. Malcovati, L. et al. Prognostic factors and life expectancy in myelodysplastic syndromes classified according to WHO criteria: a basis for clinical decision making. J. Clin. Oncol. 23, 7594-7603 (2005).

6. Lindsley, R. C. et al. Acute myeloid leukemia ontogeny is defined by distinct somatic mutations. Blood 125, 1367-1376 (2015).

7. Yoshida, K. et al. Frequent pathway mutations of splicing machinery in myelodysplasia. Nature 478, 64-69 (2011). 
8. Makishima, H. et al. Somatic SETBP1 mutations in myeloid malignancies. Nat. Genet. 45, 942-946 (2013).

9. Grossmann, V. et al. Whole-exome sequencing identifies somatic mutations of BCOR in acute myeloid leukemia with normal karyotype. Blood 118, 6153-6163 (2011).

10. Thota, S. et al. Genetic alterations of the cohesin complex genes in myeloid malignancies. Blood 124, 1790-1798 (2014).

11. Ramdzan, Z. M. \& Nepveu, A. CUX1, a haploinsufficient tumour suppressor gene overexpressed in advanced cancers. Nat. Rev. Cancer 14, 673-682 (2014).

12. Figueroa, M. E. et al. Leukemic IDH1 and IDH2 mutations result in a hypermethylation phenotype, disrupt TET2 function, and impair hematopoietic differentiation. Cancer Cell 18, 553-567 (2010).

13. Cole, C. B. et al. Haploinsufficiency for DNA methyltransferase $3 \mathrm{~A}$ predisposes hematopoietic cells to myeloid malignancies. J. Clin. Invest. 127, 3657-3674 (2017).

14. Fisher, C. L. et al. Loss-of-function additional sex combs like 1 mutations disrupt hematopoiesis but do not cause severe myelodysplasia or leukemia. Blood 115, 38-46 (2010).

15. Mori, T. et al. Somatic PHF6 mutations in 1760 cases with various myeloid neoplasms. Leukemia 30, 2270-2273 (2016).

16. Papaemmanuil, E. et al. Somatic SF3B1 mutation in myelodysplasia with ring sideroblasts. N. Engl. J. Med. 365, 1384-1395 (2011).

17. Mossner, M. et al. Mutational hierarchies in myelodysplastic syndromes dynamically adapt and evolve upon therapy response and failure. Blood 128, 1246-1259 (2016).

18. Steensma, D. P. et al. Clonal hematopoiesis of indeterminate potential and its distinction from myelodysplastic syndromes. Blood 126, 9-16 (2015).

19. Jaiswal, S. et al. Age-related clonal hematopoiesis associated with adverse outcomes. N. Engl. J. Med. 371, 2488-2498 (2014).

20. Xie, M. et al. Age-related mutations associated with clonal hematopoietic expansion and malignancies. Nat. Med. 20, 1472-1478 (2014).

21. Malcovati, L. et al. Clinical significance of somatic mutation in unexplained blood cytopenia. Blood 129, 3371-3378 (2017)

22. Sperling, A. S., Gibson, C. J. \& Ebert, B. L. The genetics of myelodysplastic syndrome: from clonal haematopoiesis to secondary leukaemia. Nat. Rev. Cancer 17, 5-19 (2017).

23. Polprasert, C. et al. Inherited and somatic defects in DDX41 in myeloid neoplasms. Cancer Cell 27, 658-670 (2015).

24. Yan, M. et al. A previously unidentified alternatively spliced isoform of $\mathrm{t}(8 ; 21)$ transcript promotes leukemogenesis. Nat. Med. 12, 945-949 (2006).

25. Ayton, P. M. \& Cleary, M. L. Molecular mechanisms of leukemogenesis mediated by MLL fusion proteins. Oncogene 20, 5695-5707 (2001).

26. Stephenson, J., Mufti, G. J. \& Yoshida, Y. Myelodysplastic syndromes: from morphology to molecular biology. Part II. The molecular genetics of myelodysplasia. Int J. Hematol. 57, 99-112 (1993).

27. Ortmann, C. A. et al. Effect of mutation order on myeloproliferative neoplasms. N. Engl. J. Med. 372, 601-612 (2015).

28. Roth, A. et al. PyClone: statistical inference of clonal population structure in cancer. Nat. Methods 11, 396-398 (2014).

29. Makishima, H. et al. Dynamics of clonal evolution in myelodysplastic syndromes. Nat. Genet. 49, 204-212 (2017).

30. Sallman, D. A. et al. Impact of TP53 mutation variant allele frequency on phenotype and outcomes in myelodysplastic syndromes. Leukemia 30, 666-673 (2016).

31. Haase, D. et al. TP53 mutation status divides myelodysplastic syndromes with complex karyotypes into distinct prognostic subgroups. Leukemia $\mathbf{3 3}$ 1747-1758 (2019).

32. Cheson, B. D. et al. Clinical application and proposal for modification of the International Working Group (IWG) response criteria in myelodysplasia. Blood 108, 419-425 (2006).

33. Bejar, R. et al. TET2 mutations predict response to hypomethylating agents in myelodysplastic syndrome patients. Blood 124, 2705-2712 (2014).

34. Genovese, G. et al. Clonal hematopoiesis and blood-cancer risk inferred from blood DNA sequence. N. Engl. J. Med. 371, 2477-2487 (2014).

35. Papaemmanuil, E. et al. Clinical and biological implications of driver mutations in myelodysplastic syndromes. Blood 122, 3616-3627 (2013)

36. Broséus, J. et al. Age, JAK2(V617F) and SF3B1 mutations are the main predicting factors for survival in refractory anaemia with ring sideroblasts and marked thrombocytosis. Leukemia 27, 1826-1831 (2013).

37. Haferlach, T. et al. Landscape of genetic lesions in 944 patients with myelodysplastic syndromes. Leukemia 28, 241-247 (2014).

38. McMahon, C. M. et al. Clonal selection with Ras pathway activation mediates secondary clinical resistance to selective FLT3 inhibition in acute myeloid leukemia. Cancer Disco. 9, 1050-1063 (2019).

39. da Silva-Coelho, P. et al. Clonal evolution in myelodysplastic syndromes. Nat. Commun. 8, 15099 (2017).

40. Agger, K. et al. UTX and JMJD3 are histone H3K27 demethylases involved in HOX gene regulation and development. Nature 449, 731-734 (2007)
41. Löwenberg, B. \& Valk, P. J. M. Molecular minimal residual disease in acute myeloid leukemia. N. Engl. J. Med. 378, 2443 (2018).

42. Bejar, R. et al. Validation of a prognostic model and the impact of mutations in patients with lower-risk myelodysplastic syndromes. J. Clin. Oncol. 30, 3376-3382 (2012)

43. Mendler, J. H. et al. RUNX1 mutations are associated with poor outcome in younger and older patients with cytogenetically normal acute myeloid leukemia and with distinct gene and MicroRNA expression signatures. J. Clin. Oncol. 30, 3109-3118 (2012).

44. Ferrando, A. A. \& López-Otín, C. Clonal evolution in leukemia. Nat. Med. 23, 1135-1145 (2017).

45. Lindsley, R. C. et al. Prognostic mutations in myelodysplastic syndrome after stem-cell transplantation. N. Engl. J. Med. 376, 536-547 (2017).

46. Kahn, J. D. et al. -truncating mutations confer resistance to chemotherapy and sensitivity to PPM1D inhibition in hematopoietic cells. Blood 132, 1095-1105 (2018).

47. Pfeilstöcker, M. et al. Time-dependent changes in mortality and transformation risk in MDS. Blood 128, 902-910 (2016)

48. Hirsch, C. M. et al. Molecular features of early onset adult myelodysplastic syndrome. Haematologica 102, 1028-1034 (2017).

49. Wang, K., Li, M. \& Hakonarson, H. ANNOVAR: functional annotation of genetic variants from high-throughput sequencing data. Nucleic Acids Res. 38, e164 (2010).

50. Sherry, S. T. et al. dbSNP: the NCBI database of genetic variation. Nucleic Acids Res. 29, 308-311 (2001).

51. Auton, A. et al. A global reference for human genetic variation. Nature 526, 68-74 (2015)

52. Lek, M. et al. Analysis of protein-coding genetic variation in 60,706 humans. Nature 536, 285-291 (2016)

53. Gonzalez Garcia, J. R. \& Meza-Espinoza, J. P. Use of the international system for human cytogenetic nomenclature (ISCN). Blood 108, 3952-3953 (2006).

54. Nagata, Y. et al. Variegated RHOA mutations in adult T-cell leukemia/ lymphoma. Blood 127, 596-604 (2016).

55. Nannya, Y. et al. A robust algorithm for copy number detection using highdensity oligonucleotide single nucleotide polymorphism genotyping arrays. Cancer Res. 65, 6071-6079 (2005).

56. Yamamoto, G. et al. Highly sensitive method for genomewide detection of allelic composition in nonpaired, primary tumor specimens by use of affymetrix single-nucleotide-polymorphism genotyping microarrays. Am. J. Hum. Genet. 81, 114-126 (2007).

57. Shah, S. P. et al. The clonal and mutational evolution spectrum of primary triple-negative breast cancers. Nature 486, 395-399 (2012).

58. Suzuki, H. et al. Mutational landscape and clonal architecture in grade II and III gliomas. Nat. Genet. 47, 458-468 (2015).

59. Coombs, C. C. et al. Therapy-related clonal hematopoiesis in patients with non-hematologic cancers is common and associated with adverse clinical outcomes. Cell Stem Cell 21, 374-382.e4 (2017).

60. Buscarlet, M. et al. DNMT3A and TET2 dominate clonal hematopoiesis and demonstrate benign phenotypes and different genetic predispositions. Blood 130, 753-762 (2017).

61. Zink, F. et al. Clonal hematopoiesis, with and without candidate driver mutations, is common in the elderly. Blood 130, 742-752 (2017).

62. Benjamini, Y. \& Hochberg, Y. Controlling the false discovery rate: a practical and powerful approach to multiple testing. J. R. Statist. Soci. B. 57, 289-300 (1995).

\section{Acknowledgements}

We are grateful to Dr Benjamin L. Ebert in Dana Farber in Dana-Farber Cancer Institute, Boston, MA for his review and thoughtful comments as to how to improve our papers. This work was supported by US National Institute of Health (NIH) grants R35 HL135795, R01HL123904, R01 HL118281, R01 HL128425, R01 HL132071 (Maciejewski), and a grant from Edward P. Evans Foundation (Maciejewski) and a grant JSPS Overseas Research Fellow (Nagata)

\section{Author contributions}

Y.N., H.M., C.K., B.P., M.A., A.G., H.A., M.A., T.K., T.R., V.A., and K.Y. performed experiments of molecular study and data analysis; H.S., T.Y., K.Y., H.T., K.C., Y.S., S. Miyano., and T.L. were committed to bioinformatics analysis of sequencing data; S. Mukherjee., A.N., M.S.,T.H., and S.O. collected specimens and were involved in planning the project; Y.N., T.L., T.R., and J.P.M. generated figures and tables, and wrote the paper Y.N. and J.P.M. lead the entire project; and all authors participated in discussions and interpretation of the data and results.

\section{Competing interests}

The authors declare no competing interests. 


\section{Additional information}

Supplementary information is available for this paper at https://doi.org/10.1038/s41467019-13001-y.

Correspondence and requests for materials should be addressed to Y.N. or J.P.M.

Peer review information Nature Communications thanks David Sallman and other anonymous,reviewer(s) for their contribution to the peer review of this work.

Reprints and permission information is available at http://www.nature.com/reprints

Publisher's note Springer Nature remains neutral with regard to jurisdictional claims in published maps and institutional affiliations. (c) (i) Open Access This article is licensed under a Creative Commons Attribution 4.0 International License, which permits use, sharing, adaptation, distribution and reproduction in any medium or format, as long as you give appropriate credit to the original author(s) and the source, provide a link to the Creative Commons license, and indicate if changes were made. The images or other third party material in this article are included in the article's Creative Commons license, unless indicated otherwise in a credit line to the material. If material is not included in the article's Creative Commons license and your intended use is not permitted by statutory regulation or exceeds the permitted use, you will need to obtain permission directly from the copyright holder. To view a copy of this license, visit http://creativecommons.org/ licenses/by/4.0/

(C) The Author(s) 2019 